OPEN ACCESS

Edited by:

Adriana Ximenes-da-Silva,

Federal University of Alagoas, Brazil

Reviewed by:

Yinghua Yu,

Xuzhou Medical University, China

Rim Hassouna,

Paris Diderot University, France

*Correspondence:

Marilia Ferreira Frazão Tavares de

Melo

mariliafrazao@hotmail.com

Specialty section:

This article was submitted to Neuroenergetics, Nutrition and Brain

Health,

a section of the journal

Frontiers in Neuroscience

Received: 01 October 2018

Accepted: 07 January 2019

Published: 23 January 2019

Citation:

Melo MFFT, Pereira DE, Moura RL, Silva EB, Melo FALT, Dias CCQ, Silva MCA, Oliveira MEG, Viera VB, Pintado

MME, Santos SG and Soares JKB

(2019) Maternal Supplementation

With Avocado (Persea americana Mill.) Pulp and Oil Alters Reflex Maturation,

Physical Development, and Offspring

Memory in Rats.

Front. Neurosci. 13:9.

doi: 10.3389/fnins.2019.00009

\section{Maternal Supplementation With Avocado (Persea americana Mill.) Pulp and Oil Alters Reflex Maturation, Physical Development, and Offspring Memory in Rats}

\author{
Marilia Ferreira Frazão Tavares de Melo ${ }^{1,2 *}$, Diego Elias Pereira ${ }^{1,2}$, \\ Renally de Lima Moura ${ }^{2}$, Elisiane Beatriz da Silva ${ }^{2}$, Flávio Augusto Lyra Tavares de Melo ${ }^{3}$, \\ Celina de Castro Querino Dias ${ }^{1,2}$, Maciel da Costa Alves Silva ${ }^{2}$, \\ Maria Elieidy Gomes de Oliveira ${ }^{1,4}$, Vanessa Bordin Viera ${ }^{5}$, \\ Maria Manuela Estevez Pintado ${ }^{6}$, Sócrates Golzio dos Santos ${ }^{3}$ and \\ Juliana Késsia Barbosa Soares ${ }^{1,2}$

\begin{abstract}
${ }^{1}$ Program of Food Science and Tecnology, Universidade Federal da Paraíba, João Pessoa, Brazil, ${ }^{2}$ Laboratory of Experimental Nutrition, Department of Nutrition, Universidade Federal de Campina Grande, Cuité, Brazil, ${ }^{3}$ Universidade Paraíba, João Pessoa, Brazil, ${ }^{5}$ Laboratory of Bromatology, Department of Nutrition, Universidade Federal de Campina Grande, Cuité, Brazil, ${ }^{6}$ Center for Biotechnology and Chemistry, School of Biotechnology, Catholic University of Porto, Porto, Portugal
\end{abstract} \\ Federal da Paraíba, João Pessoa, Brazil, ${ }^{4}$ Laboratory of Bromatology, Department of Nutrition, Universidade Federal da
}

Avocado (Persea americana Mill.) is an oleaginous fruit source of fatty acids with high levels of neuroprotective phytocomplexes. The objective of this study was to evaluate the development of reflex and somatic maturation, fatty acid profiles in the brain, and memory in different stages of life in the offspring of dams supplemented with avocado pulp and oil during gestation and lactation. The dams were randomly divided into three groups ( $n=15 \mathrm{pups} /$ group), and recieved by gavage supplementation: control group (CG)-distilled water; Avocado Oil (AO)-3,000 mg avocado oil/kg animal weight, and Avocado Pulp (AP)-3,000 mg avocado pulp/kg animal weight. We performed the following tests: Analysis of Somatic Development and Ontogeny of Postnatal Reflex (TO to T21), the Open Field Habituation Test and the Object Recognition Test (ORT) in the adolescent (T45) and adult (T90) phases. The cerebral fatty acids content was evaluated at times T0, T21, T45, and T90. The results were analyzed using the statistical program GraphPad Prism and significant statistics were considered when $p<0.05$. Acceleration of reflex maturation and reflex ontogeny was observed in the offspring of $\mathrm{AO}$ and AP fed dams, with the results being more pronounced in the pulp fed group $(p<0.05)$. All groups presented a decrease in the ambulation parameter in the second exposure to the Open Field Habituation Test, at T45 and T90 ( $p<0.05)$. In the ORT, the $\mathrm{AO}$ and AP offspring presented memory improvements in the short and long term in the adult and adolescent phases $(p<0.05)$. The results of the brain fatty acid profiles presented higher polyunsaturated fatty acids (PUFA) content in the AO and AP groups at T21, T45, and T90. The docosahexaenoic fatty acid (DHA) content was higher at T21 
(AO and AP), at T45 (AO and AP), and at T90 (AP) $(p<0.05)$. The arachidonic acid (ARA) content was higher at T45 (AO and AP), and at T90 (AO) $(p<0.05)$. Maternal supplementation with avocado oil and pulp anticipates reflex maturation and somatic postnatal development, and improves memory during the adolescent and adult phases.

Keywords: avocado, cerebral fatty acids, postnatal development, memory, rats

\section{INTRODUCTION}

Adequate fetal and postnatal development is influenced by maternal nutrition (Brenna and Lapillonne, 2009; Mennitti et al., 2015). During this period, considered developmentally critical, lipids are essential to tissue construction and determination of body growth (Morgane et al., 1993; Herrera and OrtegaSenovilla, 2014). Lipids structurally compose the nervous system, stimulate its development and differentiation, and even regulate neuronal cell migration (González and Visentin, 2016; Prado et al., 2018).

The quality of lipids in the diet during gestation and lactation determines the type of fatty acid (FA) that will accumulate in the fetal tissue through placental transfer and through the breast milk after birth (Lauritzen and Carlson, 2011; Innis, 2014). Fatty acids are essential nutrients for the development and maintenance of brain functions and are closely related to learning processes and memory. They demonstrate a positive correlation to neurodevelopment in the offspring through maternal lipid intake (Apryatin et al., 2017; Melo et al., 2017; Pase et al., 2017).

The principal FAs involved in brain development are polyunsaturated fatty acids (PUFAs): linoleic acid (C18: $2 \omega$ 6) (LA), $\alpha$-linolenic acid (C18: $3 \omega$-3) (ALA), arachidonic acid (ARA; 20:4 $\omega-6$ ), docosahexaenoic acid (DHA; 22:6 $\omega-3$ ), and eicosapentaenoic acid (EPA, 20:5, $\omega-3$ ) (Makrides et al., 2011; González and Visentin, 2016). Since they are not endogenously synthesized, they are considered essential, and their aquisition occurs only through dietary intake of sources rich in endogenous precursors; ALA and LA (Sinclair, 1975). FA accumulating in brain tissue actively participates in the formation of neuronal membranes (Yehuda, 2012), improving learning, and memory and increasing synaptic and neurogenic plasticity (Dyall, 2017). The influence of maternal PUFAs on the development of reflexes has been evaluated in experimental studies with the offspring (Souza et al., 2012).

Non-essential FA, such as oleic monounsaturated fatty acid (18: $1 \omega-9)$ and palmitic saturated (16: $0 \omega-7)$, can be endogenously synthesized and also transferred through the placenta during gestation; secreted into the maternal milk and accumulate in the brain and other organs during fetal development (Innis, 2004, 2005). Oleic fatty acid is one of the main constituents of myelin (Garbay et al., 2000); it is related to axonal growth and neuronal grouping (Medina and Tabernero, 2002). Palmitic fatty acid participates in the processes of palmitoylation, gliogenesis, synaptogenesis, and myelination (González and Visentin, 2016).

Several sources of fatty acids can be used for maternal supplementation. The avocado (Persea americana Mill.) is an oleaginous fruit that has thus aroused scientific interest. Its lipidic composition includes monounsaturated oleic fatty acid ( $\omega-9)$, saturated palmitic ( $\omega-7)$, and two linoleic polyunsaturates; $(\omega-6)$, and $(\omega-3)$ at lower levels (USDA, 2011; Dreher and Davenport, 2013). Avocado is also a source of neuroprotective antioxidant phytocomplexes (phytosterols, carotenoids, flavonoids) (Ameer, 2016).

Considering associations between maternal lipid consumption and its effects on the neurodevelopment of the offspring and the scarcity of information in the literature on the effect of avocado consumption at this stage, we hypothesized that maternal supplementation with avocado might anticipate the appearance of the reflexes and somatic maturation, and improve the offspring's memory. The objective of this research was to evaluate the offspring of dams supplemented with avocado oil and pulp during gestation and lactation for somatic and reflex development, analyze fatty acid profiles in the brain, and memory function through adulthood.

\section{MATERIALS AND METHODS}

\section{Avocado}

Avocado (Persea americana Mill.) of the Hass variety was obtained from the commercial producer: Fazenda Jaguacy Avocado Brasil ${ }^{\circledR}$, located in the municipality of Bauru, São Paulo: latitude $22^{\circ} 19^{\prime} 18^{\prime \prime} \mathrm{S}$, longitude $49^{\circ} 04^{\prime} 13^{\prime \prime} \mathrm{W}$, and $526 \mathrm{~m}$ altitude. Part of the fruit was used to extract oil and another part was lyophilized to obtain pulp powder. The lyophilized powder was vacuum packed, and stored at $-20^{\circ} \mathrm{C}$. The oil and pulp were offered by gavage starting on the seventh day of gestation and throughout the lactation period until the 21st postnatal day.

\section{Analysis of Fatty Acid Composition in Avocado Oil and Pulp}

The fatty acid profiles of the oil and pulp were analyzed (Folch et al., 1957; Hartman and Lago, 1973) (Table 1).

\section{Lipidic Extraction}

Sample were weighed ( $2 \mathrm{~g}$ of each) in a beaker and added to $30 \mathrm{ml}$ of chloroform:methanol mixture (2:1). After this addition, the content was transferred to a deep glass container with the side covered with aluminum foil and stirred for $2 \mathrm{~min}$ with the help of grinder. The triturate was filtered through qualitative filter paper into a $100 \mathrm{ml}$ graduated cylinder with a polished mouth. Next, the vessel walls were washed with an additional $10 \mathrm{~mL}$ of chloroform:methanol which was also filtered with the previous volume. The volume of the filtered extract of the graduated cylinder was recorded with the graduated cylinder closed. Twenty 
TABLE 1 | Fatty acid composition of avocado oil and lyophilized pulp (Persea americana Mill.): hass variety.

\begin{tabular}{|c|c|c|c|}
\hline & & Avocado oil & Avocado pulp \\
\hline \multicolumn{2}{|c|}{ Acids Fat } & \multicolumn{2}{|c|}{$100 \mathrm{~g}^{-1}$ lipids } \\
\hline \multicolumn{4}{|l|}{ SATURATED } \\
\hline Palmitic acid & C16:0 & 22.80 & 22.41 \\
\hline Stearic acid & C18:0 & 0.60 & 0.64 \\
\hline Araquidic acid & C20:0 & 0.07 & 0.06 \\
\hline Lignoceric acid & C20:4 & 0.07 & 0.08 \\
\hline \multicolumn{2}{|c|}{$\sum$ SFA } & 23.54 & 23.19 \\
\hline \multicolumn{4}{|l|}{ MONOUNSATURED } \\
\hline Palmitoleic acid & $\mathrm{C} 16: 1 \omega-7$ & 12.98 & 13.40 \\
\hline Heptadeacenoic acid & $C 17: 1 \omega-7$ & 0.10 & 0.09 \\
\hline Oleic acid & 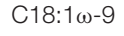 & 45.92 & 41.66 \\
\hline Gondoic acid & C20: $1 \omega-9$ & 0.16 & 0.14 \\
\hline \multicolumn{2}{|c|}{$\sum M U F A$} & 59.16 & 55.29 \\
\hline \multicolumn{4}{|l|}{ POLYUNSATURED } \\
\hline Linoleic acid & 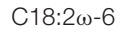 & 12.10 & 13.11 \\
\hline$\alpha$-linolenic acid & 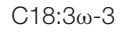 & 0.72 & 0.81 \\
\hline \multicolumn{2}{|c|}{$\sum$ PUFA } & 12.82 & 13.93 \\
\hline
\end{tabular}

percentage of the final volume of the filtered extract was added to $1.5 \%$ sodium sulfate. The mixture was stirred with the graduated cylinder closed and given time for the phases to separate. It was observed that the upper phase was $\sim 40 \%$ and the bottom $60 \%$ of the total volume. The volume of the lower phase was recorded and then the upper phase was discarded by suction with a graduated pipette. For lipid quantification, an extracted aliquot of $5 \mathrm{~mL}$ (lower phase) was separated with a volumetric pipette and transferred to a previously weighed beaker. This beaker was placed in an oven at $105^{\circ} \mathrm{C}$ so the solvent mixture could evaporate, being careful that the fat would not be degraded by heat. After cooling in a desiccator, the beaker was weighed and the fat residue weight was obtained from the difference (Folch et al., 1957).

\section{Transesterification of Fatty Acids}

In the sample treatment, methylation of fatty acids present in the lipid extract was carried out following the methodology described by Hartman and Lago (1973). An aliquot of the lipid extract was taken, calculated for each sample according to the fat conte2nt found in the lipid measurement, and performed according to the (Folch et al., 1957), adding $1 \mathrm{ml}$ of internal standard (C19:0) and a saponification $(\mathrm{KOH})$ solution. This solution was subsequently brought to heating under reflux for $4 \mathrm{~min}$. Esterification solution was added immediately after, returning the solution to heating under reflux for 3 more minutes. Next, the sample was allowed to cool before subsequent washings with ether, hexane and distilled water, finally obtaining an extract (with the methyl esters and solvents), which was conditioned into a properly identified amber glass until complete drying of the solvents. After drying, a suspension in $1 \mathrm{ml}$ of hexane was made and packaged into a vial for further chromatographic analysis. The aliquots of saponification and esterification solutions were determined according to the methodology described by Hartman and Lago (1973).

\section{Gas Chromatography Analysis}

A gas chromatograph (VARIAN 430-GC, California, EUA), coupled to a capillary column of fused silica (CP WAX 52 $\mathrm{CB}$, VARIAN, California, EUA) with dimensions of $60 \mathrm{~m} \times$ $0.25 \mathrm{~mm}$ and $0.25 \mathrm{~mm}$ film thickness was used with helium as carrier gas (Flow rate of $1 \mathrm{ml} / \mathrm{min}$ ). The initial oven temperature was $100^{\circ} \mathrm{C}$ programmed to reach $240^{\circ} \mathrm{C}$, increasing $2.5^{\circ} \mathrm{C}$ per minute for $30 \mathrm{~min}$, totaling $86 \mathrm{~min}$. The injector temperature was maintained at $250^{\circ} \mathrm{C}$ and the detector at $260^{\circ} \mathrm{C} .1 .0 \mu \mathrm{l}$ aliquots of esterified extract were injected in a Split/Splitless injector. The chromatograms were recorded using Galaxie Chromatography Data System software. The fatty acids results were quantified by integration the areas of the methyl esters and are expressed in percentage by area.

\section{Analysis of Antioxidant Content of Oil and Lyophilized Avocado Pulp}

The oil and pulp were analyzed for their total phenolic, flavonoid, and carotenoid components. The antioxidant capacity was also analyzed using the ABTS, FRAP, and $\mathrm{IC}_{50}$ methods.

\section{Extraction}

Avocado pulp constituents were extracted with both 80:20 EtOH: $\mathrm{H}_{2} \mathrm{O} \mathrm{v} / \mathrm{v}$ and evaluated for ABTS scavenging capacity, ferric reducing activity (FRAP) and total flavonoids. For total phenolic contents $100 \% \mathrm{MeOH}$. Oil constituents were extracted with both 80:20 MeOH:H2O v/v and evaluated for FRAP, ABTS, total phenolic and flavonoids contents. All the extractions were performed in triplicate.

\section{Determination of Total Phenolic Compounds (TPC)}

In order to estimate the total phenolic compounds, the methodology described by Liu et al. (2002) was used with minor modifications. The absorbance of the extract was compared with a gallic acid standard curve for estimating concentration of TPC in the sample. The TPC was expressed as mg of gallic acid equivalents (GAE) per $100 \mathrm{~g}$ of avocado oil and pulp on the basis of dry weight (DW).

\section{Determination of Total Flavonoids}

The total flavonoid content was measured using the colorimetric assay developed by Zhishen et al. (1999). The absorbance of the extract was compared with a catechin standard curve for estimating concentration of flavonoids contents in the sample. The flavonoids contents was expressed as $\mathrm{mg}$ of catechin equivalents (QE) per $100 \mathrm{~g}$ of avocado oil and pulp on the basis of dry weight (DW).

\section{Antioxidant Activity-FRAP Method}

The FRAP method was performed according to Benzie and Strain (1999), with modifications proposed by Pulido et al. (2000). The FRAP solution was used as reference reagent, and absorbance was read at $593 \mathrm{~nm}$. The results were expressed in $\mu$ mol of trolox equivalents per gram of avocado pulp on dry weight (DW) basis $\left(\mu \mathrm{mol} \mathrm{TE} / \mathrm{g}^{-1}\right)$. 


\section{Antioxidant Activity-ABTS Method ${ }^{+}$}

The ABTS method was carried out according to the methodology described by Surveswaran et al. (2007), with modifications. The results were expressed in $\mu \mathrm{mol}$ of trolox equivalent per gram of avocado oil and pulp on dry weight (DW) basis ( $\mu \mathrm{mol}$ $\left.\mathrm{TE} / \mathrm{g}^{-1}\right)$. Where $\mathrm{A}_{0}$ is the absorbance of the control and as is the absorbance of the sample. The effective concentration had $50 \%$ radical inhibition activity $\left(\mathrm{IC}_{50}\right)$, expressed as mg extract/ $\mathrm{mL}$, which was determined from the graph of the free radical scavenging activity (\%) against the extract concentration.

The pulp and oil, respectively, presented total phenolic contents of 64.61 and $49.50 \mathrm{mg}$ GAE/100 g, total flavonoids of 39.38 and $33.75 \mathrm{mg} \mathrm{CE} / 100 \mathrm{~g}$, and total carotenoids of 87.00 and $9.87 \mathrm{mg} / 100 \mathrm{~g}$. For antioxidant activity, the pulp and oil presented respective FRAP values of 0.08 and $0.03 \mu \mathrm{mol} \mathrm{TE} / \mathrm{g}, \mathrm{ABTS}$ of 2.02 and $0.17 \mu \mathrm{mol} \mathrm{TE} / \mathrm{g}$, and $\mathrm{IC}_{50}$ of 59.86 and $443.99 \mathrm{mg} / \mathrm{mL}$.

\section{Animals and Experimental Groups}

Females of the Wistar lineage (90 days old/weights $250 \pm 50 \mathrm{~g}$ ) were obtained from the Laboratory of Experimental Nutrition, at the Federal University of Campina Grande-LANEX/UFCG and were breeded to obtain 45 newborn rats. The females were mated while maintained at the ratio of two females to each male. After confirmation of pregnancy, the rats were housed in individual polypropylene maternity cages $(60 \mathrm{~cm}$ in length, $50 \mathrm{~cm}$ wide, and $22 \mathrm{~cm}$ in height), under standard laboratory conditions (temperature $22 \pm 1{ }^{\circ} \mathrm{C}$, humidity $65 \pm 5 \%$, light/dark cycle of 12/12 h-artificial light from 6:00 to 18:00).

To obtain the offspring, 24 (Folch et al., 1957) female rats were randomly divided into three groups ( $n=15$ pups for each group): Control (CG)-supplemented with distilled water; Avocado Oil (AO)-supplemented with $3,000 \mathrm{mg}$ of avocado oil/kg of animal weight; and Avocado Pulp (AP)-supplemented with $3,000 \mathrm{mg}$ of avocado pulp/ $\mathrm{kg}$ of animal weight. Gavage was administered from the 7 th day of gestation until the 21st day of lactation: Standard feed (Presence Purina ${ }^{\circledR}$, São Paulo, Brazil) and water was offered ad libitum. After weaning, the offspring received standard ration until adulthood. The research followed an experimental protocol in accordance with the ethical recommendations of the National Institute of Health (Bethesda,
USA), and was approved by the ethics research committee of the Federal University of Campina Grande No: 006/2017 and avocado registered in SisGen $n^{\circ}$ A737D56.

\section{Experimental Procedures}

The neonates were weighed and evaluated for reflex ontogenesis and somatic development parameters each day from birth until weaning. For fatty acid content analysis, brains were collected on the first day of life (T0), on weaning day (T21), at adolescence (T45), and as adults (T90). The memory evaluation tests were performed in adolescence and adulthood. The experimental protocol is detailed in Figure 1.

\section{Removal of Brains and Fatty Acid Content Analysis}

At T0, after sexing and manipulation for litter reduction, surplus puppies were randomly chosen for removal of the brain, which was removed upon decapitation. At T21, T45, and T90 brains were also removed and stored at $-20^{\circ} \mathrm{C}$ until the day of analysis and quantification of fatty acid content $(n=6)$.

The fatty acid profile of the brains was determined using the (Hartman and Lago, 1973) method with transesterification and subsequent identification by gas chromatography (Varian 430GC).

\section{Reflex Ontogeny and Somatic Response}

Each day, from the 1st to the 21st day of life at from between 06:00 to 8:00 a.m. in the morning, somatic responses and reflex ontogeny were evaluated. The response was considered consolidated when the expected reaction was repeated for three consecutive days, being the 1st day of the appearance considered as the day of consolidation. The daily observation time for each parameter was $10 \mathrm{~s}$. The reflex study followed the experimental model established by Smart and Dobbing (1971) (Table 2). Somatic maturation indicators were also evaluated: Aural "Pavilion" Opening (APO), Auditory Conduit Opening (ACO), Eye Opening (EO), Eruption of Upper Incisive Teeth (EUIT) and Inferior Teeth (EIIT), Appearance of Epidermal Hair $(\mathrm{AEH})$, and Tail Length (TL).

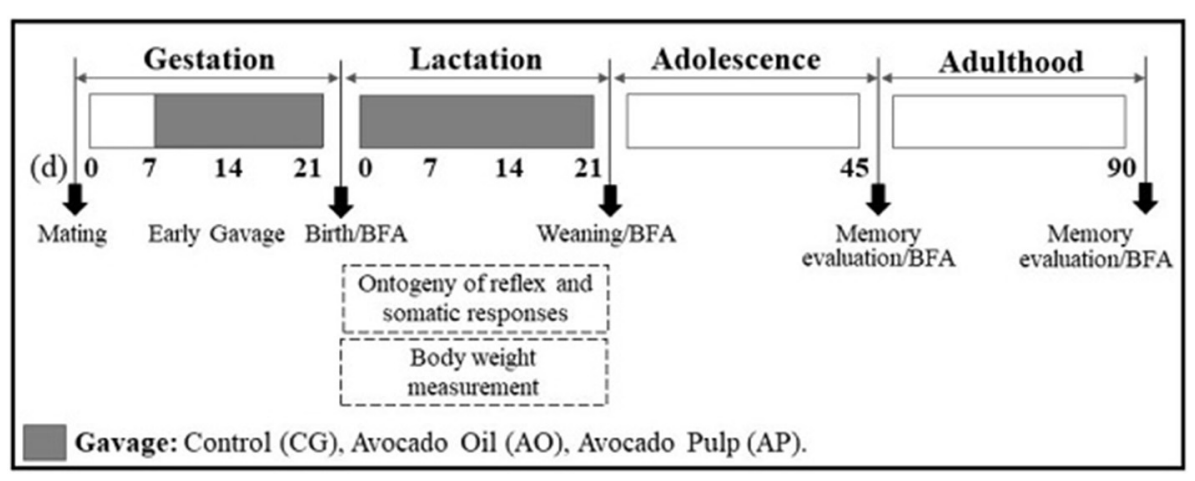

FIGURE 1 | Experimental protocol. Sequence of experimental days conducted with Wistar rats supplemented during gestation and lactation, and of their offspring. (d): day; BFA: brain fatty acids. 
TABLE 2 | Description of the reflex test.

\begin{tabular}{|c|c|c|}
\hline Reflex & Stimulus & Response \\
\hline Palmar grasp (PG) & $\begin{array}{l}\text { Light percussion on the } \\
\text { palm of the right foreleg. }\end{array}$ & Quick bending of ankles. \\
\hline Righting reflex (RR) & $\begin{array}{l}\text { The rat is placed in supine } \\
\text { position on a surface. }\end{array}$ & $\begin{array}{l}\text { Return to the prone position } \\
\text { with all paws in } 10 \mathrm{~s} .\end{array}$ \\
\hline $\begin{array}{l}\text { Cliff avoidance } \\
\text { (CA) }\end{array}$ & $\begin{array}{l}\text { The rat is placed on a flat } \\
\text { and high surface (table), with } \\
\text { legs toward the extremity. }\end{array}$ & $\begin{array}{l}\text { Moves to one side and } \\
\text { walks in the opposite } \\
\text { direction to the edge. }\end{array}$ \\
\hline $\begin{array}{l}\text { Vibrissa placing } \\
\text { (VP) }\end{array}$ & $\begin{array}{l}\text { The animal is suspended by } \\
\text { the tail and its vibrissae } \\
\text { lightly touch the edge of a } \\
\text { flat surface. }\end{array}$ & $\begin{array}{l}\text { Both front legs are placed } \\
\text { on the table, performing } \\
\text { march movements. }\end{array}$ \\
\hline $\begin{array}{l}\text { Negative geotaxis } \\
\text { (GN) }\end{array}$ & $\begin{array}{l}\text { The rat is placed at the } \\
\text { center of an inclined ramp } \\
\text { with head facing } \\
\text { downwards. }\end{array}$ & $\begin{array}{l}\text { Body spin at an angle of } \\
180^{\circ} \text {, positioning head } \\
\text { upwards. }\end{array}$ \\
\hline $\begin{array}{l}\text { Auditory startle } \\
\text { response (AS) }\end{array}$ & $\begin{array}{l}\text { Intense and sudden sound } \\
\text { stimulus. }\end{array}$ & $\begin{array}{l}\text { Retraction of anterior and } \\
\text { posterior legs, with rapid } \\
\text { and involuntary body } \\
\text { immobilization. }\end{array}$ \\
\hline $\begin{array}{l}\text { Free-fall righting } \\
\text { (FFR) }\end{array}$ & $\begin{array}{l}\text { Held by four legs at a height } \\
\text { of } 30 \mathrm{~cm} \text {, it is released in } \\
\text { free fall on a synthetic foam } \\
\text { bed. }\end{array}$ & $\begin{array}{l}\text { Position recovery during } \\
\text { freefall on the surface } \\
\text { supported by four paws. }\end{array}$ \\
\hline
\end{tabular}

\section{Memory Evaluation Tests Open Field Habituation Test}

During adolescent phase and adulthood the animals were submitted to the Open Field Habituation test and the Object Recognition Test (ORT). Each animal was exposed to the open field twice, in the first stage, the habituation test was performed; and after 7 (seven) days, the same test was repeated in order to compare the locomotor activity of the animals for evaluation of non-associative learning (Rachetti et al., 2012). The parameter analyzed through this test is the amount of explorative interactions taken by the animal to the field, considering the locomotion of the four legs toward the interior of each field. The test observation time was $10 \mathrm{~min}$. The procedure was performed between 06:00 and 08:00 a.m., on each test day, and the sessions were filmed with a video camera. For each animal tested, the apparatus was cleaned before starting, and after completion of the test with a $10 \%$ alcohol solution.

\section{Object Recognition Test (ORT)}

To evaluate the short and long term memory, the Object Recognition Task (ORT) was used. The test was performed in the open field apparatus $(60 \times 60 \times 60 \mathrm{~cm})$, colored black, with six lines crossing forming 6-20 $\times 20 \mathrm{~cm}$ quadrants, uniformly lit, and with black color objects, with different shapes (rectangular or pyramid), and textures (smooth or rough) (Nava-Mesa et al., 2013).

The test consisted of 4 (four) 10 min trials, taking place in 3 (three) steps: (1) Day 1-habituation for $10 \mathrm{~min}$ to minimize manipulation stress; (2) Day 2-performed $24 \mathrm{~h}$ after the habituation test, where each animal was placed in the open field containing two objects (FO1 and FO2) with identical textures (smooth), but with different forms (triangle and prismatic rectangle), located in two randomly chosen opposite corners. On the same day, yet $1 \mathrm{~h}$ later, the animal was placed in the open field again to explore two objects (FO1 in its original location, and a new object-NO1, identical to FO1 but with a different texture, and located in the place where $\mathrm{FO} 2$ had been placed during the habituation test; and (3) Day 3-was performed $24 \mathrm{~h}$ after the short duration test; each animal was placed in the open field to explore two objects (FO2 in its original place) and a new object (NO2) being identical to $\mathrm{FO} 2$ but with different texture (Figure 2).

To evaluate short-term memory, the time spent by the animal in exploring the new differently textured object (NO1) was observed. To evaluate the long-term memory, the time spent by the animal in exploring the new differently textured object (NO2) was observed at $24 \mathrm{~h}$ after the first exploration, on day 2 . The sessions were filmed with a video camera and for each animal tested; the device was cleaned with $10 \%$ alcohol before starting and after the test. The results for the exploration times were calculated for each animal and expressed by the ratio $\mathrm{TN} /(\mathrm{TF}+$ $\mathrm{TN}$ ) $\mathrm{TN}=$ time spent exploring the new object; $\mathrm{TF}=$ time spent exploring the familiar object (Gustavsson et al., 2010; D'avila et al., 2017).

\section{Statistical Analysis}

The results of the evaluation of reflex ontogeny and somatic development were expressed as median values for the day (MinMax), and analyzed by Kruskal-Wallis variance analysis followed by Dunn's test $(p<0.05)$. Other results were expressed as mean \pm SEM, and analyzed by ANOVA followed by Tukey $(p<0.05)$. The statistical program GraphPad Prism was used.

\section{RESULTS}

\section{Composition of Fatty Acids in Brains of Offspring After Birth}

The composition of saturated fatty acids in the AO group offspring brains on the first day of life presented reduced myristic, palmitic, and stearic fatty acids as compared to the CG and AP groups ( $p<0.05)$; the AP group offspring presented lower levels of myristic and higher levels of palmitic fatty acids as compared to CG offspring $(p<0.05)$ (Table 3 ).

Palmitoleic, vaccenic e oleic (monounsaturates) were also found decreased in the $\mathrm{AO}$ group offspring as compared to the CG and AP group offspring $(p<0.05)$. However, the AP groups presented higher values for these fatty acids then the CG and AO $(p<0.05)$ (Table 3).

The total PUFA content was $15 \%$ lower in the AO group offspring (10\% lower in the AP group) as compared to the $\mathrm{CG}$ offspring. The $\mathrm{AO}$ offspring presented reductions in linoleic, eicosadienoic, arachidonic, docosatetraenoic, and docosahexaenoic polyunsaturated fatty acids as compared to the CG and AP offspring $(p<0.05)$. AP offspring also presented reductions in linoleic, eicosadienoico, docosatetraenoic, and docosahexaenoic acids as compared to the CG offspring $(p<0.05)$. However, eicosatrienoic and 


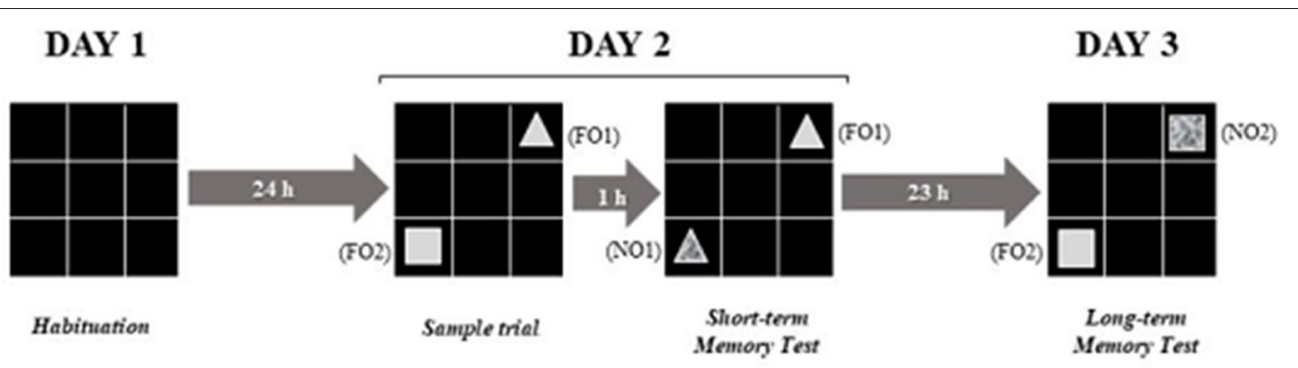

FIGURE 2 | Experimental design adapted from the object recognition test. Source: adaptation of Nava-Mesa et al. (2013).

docosapentaenoic acid levels were higher in the AP and AO offspring brains compared to the CG offspring $(p<0.05)$ (Table 3).

\section{Composition of Fatty Acids in Offspring Brains at the end of Lactation (21 Days of Life)}

At 21 days of age, myristic, palmitic, stearic, and behenic saturated fatty acids levels were found decreased in the AO and AP group offspring brains when compared to the CG $(p<0.05)$. The AP group presented higher levels of these fatty acids than the AO group $(p<0.05)$ (Table 3 ).

Both AO and AP groups presented lower total MUFA values, with reductions in palmitoleic, vaccenic, oleic, and erucic fatty acids in AO brains compared to the CG. For palmitoleic and oleic fatty acids, the AP group brains also presented lower total values as compared to the CG and $(p<0.05)$. Gondoic acid alone was higher in the AP group as compared to the CG $(p<0.05)$ (Table 3).

The polyunsaturates (linoleic, eicosadienoic, arachidonic, and docosatetraenoic acid) were decreased in the AO and AP brains as compared to the CG $(p<0.05)$. However, total PUFAs were, respectively, 13.5 and $28 \%$ higher in the AO and AP groups as compared to the CG; due to the increased DHA and docosapentaenoic acid levels. Also the total PUFAs were higher in the AP group when compared to the AO group $(p<0.05)$ (Table 3).

\section{Composition of Fatty Acids in Offsprings' Brains in Adolescence (45 Days of Life)}

Saturated fatty acid levels in the offspring brains (adolescents) were similar for all groups; except for behenic acid, which was higher in the AO and AP groups as when compared to the CG $(p<0.05)$ (Table 4).

There was no difference for monounsaturated acid contents. However, total PUFAs were $48.85 \%$ higher in the AO brains and $54.77 \%$ in the AP brains than in the CG brains. Compared to the CG brains, increased levels of DHA and docosapentaenoic fatty acid were found in the AO brains; and arachidonic, and docosahexaenoic acids were higher in the PA brains $(p<0.05)$ (Table 4).

\section{Composition of Fatty Acids in the Adult Offspring Brain (90 Days of Life)}

In adulthood, the content of saturated palmitic, stearic and behenic fatty acids in the $\mathrm{AO}$ offspring group brains was higher than the AP or control groups $(p<0.05)$. In relation to monounsaturated fatty acids, vacênic acid was different between the groups, with higher levels in the $\mathrm{AO}$ and $\mathrm{AP}$ brains as compared to the CG $(p<0.05)$. Monounsaturate erucic acid was higher in the AO brain as compared to the AP group and the controls $(p<0.05)$ (Table 4).

Linoleic, eicosadienoic and eicosatrienoic polyunsaturates presented higher levels in the AP offspring than in the AP and CG offspring $(p<0.05)$. Arachidonic acid was higher in the AO groups as compared to the CG, and docosahexaenoic acid presented higher levels in the AP group as compared to the AO and control groups $(p<0.05)$ (Table 5). Total PUFAs were higher in the brains of the AP (22\%) groups as compared to the controls (Table 4).

\section{Body Weight and Tail Length}

The body weight results for offspring of mothers treated with avocado oil and pulp during gestation and lactation are shown in Figure 3. The weights of the offspring of the pulp group (AP) were significantly lower than the control group (CG) during the first week of lactation (1st and 7th day), and when compared to the oil group ( $\mathrm{AO})$, the weights were lower from the 7 th to the 21 st day $(p<0.05)$. Only on the 14th day of lactation did the AO pups present significantly higher weights as compared to the CG $(p<0.05)$. By the end of lactation, the differences differences did not persist.

The tail lengths presented significant differences only on the first day of life, where the AP pupils presented larger sizes as compared to the AO group $(p<0.05)$ (Figure 4$)$. The difference did not remain beyond the 7 th day (through the end of lactation).

\section{Ontogenesis of Reflex, and Somatic Maturation}

The offspring of mothers supplemented with pulp (AP) compared to the CG presented early disappearance of the PG, and appearance of the following reflexes: VP, CA, GN, AS, and FFR $(p<0.05)$. These same pups also anticipated the VP, GN, and AS reflexes as compared to the AO group $(p<0.05)$. The 
TABLE 3 | Composition of fatty acids present in the brain puppies (T0 and T21) of dams supplemented with oil and avocado pulp.

\begin{tabular}{|c|c|c|c|c|c|c|}
\hline \multirow[b]{3}{*}{ Fatty acids } & \multicolumn{6}{|c|}{ Groups } \\
\hline & \multicolumn{3}{|c|}{ Brain-T0 day of life } & \multicolumn{3}{|c|}{ Brain-T21 day of life } \\
\hline & CG & AO & AP & CG & AO & AP \\
\hline \multicolumn{7}{|l|}{ SATURATED } \\
\hline Myristic acid C14:0 & $1.51 \pm 0.05$ & $1.15 \pm 0.04^{*}$ & $1.40 \pm 0.05^{\star \#}$ & $0.40 \pm 0.00$ & $0.32 \pm 0.01^{*}$ & $0.43 \pm 0.11^{\star \#}$ \\
\hline Palmitic acid C16:0 & $25.27 \pm 0.30$ & $22.57 \pm 0.20^{*}$ & $26.16 \pm 0.40^{\star \#}$ & $20.11 \pm 0.05$ & $17.58 \pm 0.07^{\star}$ & $18.88 \pm 1.01^{\star \# \#}$ \\
\hline Stearic acid C18:0 & $15.63 \pm 0.10$ & $13.40 \pm 0.04^{*}$ & $15.01 \pm 0.09^{\star \#}$ & $17.90 \pm 0.50$ & $15.16 \pm 0.64^{\star}$ & $15.92 \pm 0.84^{\star \#}$ \\
\hline Behenic acid C22:0 & - & - & - & $0.16 \pm 0.01$ & $0.13 \pm 0.02^{*}$ & $0.12 \pm 0.01^{\star}$ \\
\hline Total & 42.41 & $37.12^{*}$ & $42.57^{\star}$ & 38.57 & $33.19^{*}$ & $35.35^{\star \#}$ \\
\hline \multicolumn{7}{|l|}{ MONOUNSATURATED } \\
\hline Palmitoleic acid $\mathbf{C} 16: 1 \omega 7 \mathrm{c}$ & $3.74 \pm 0.03$ & $1.48 \pm 0.02^{\star}$ & $3.93 \pm 0.03^{\star \#}$ & $0.69 \pm 0.00$ & $0.47 \pm 0.01^{*}$ & $0.39 \pm 0.02^{\star \#}$ \\
\hline Vaccenic acid C18:1 $\omega 7 \mathrm{c}$ & $2.90 \pm 0.02$ & $2.63 \pm 0.03^{*}$ & $3.03 \pm 0.01^{\star \#}$ & $2.91 \pm 0.10$ & $2.54 \pm 0.18^{\star}$ & $2.76 \pm 0.22^{\#}$ \\
\hline Oleic acid C18:1 $\omega \mathbf{9}$ & $11.12 \pm 0.10$ & $9.86 \pm 0.09^{*}$ & $11.41 \pm 0.10^{\star \#}$ & $12.96 \pm 0.20$ & $10.83 \pm 0.26^{*}$ & $11.74 \pm 1.19^{\star \#}$ \\
\hline Gondoic acid C20:1 $1 \omega 9$ & $0.20 \pm 0.02$ & $0.22 \pm 0.01^{*}$ & $0.20 \pm 0.02^{\#}$ & $0.52 \pm 0.04$ & $0.60 \pm 0.06$ & $0.67 \pm 0.12^{\star}$ \\
\hline Erucid acid C22:1 $\mathbf{\omega 9}$ & - & - & - & $0.09 \pm 0.01$ & $0.06 \pm 0.01^{*}$ & $0.07 \pm 0.03$ \\
\hline Total & 17.96 & $14.19^{*}$ & $18.57^{\star \#}$ & 17.17 & $14.50^{\star}$ & $15.56^{\star \#}$ \\
\hline \multicolumn{7}{|l|}{ POLYUNSATURATED } \\
\hline Linoleic acid C18:2 $\omega 6 \mathrm{c}$ & $1.04 \pm 0.10$ & $0.78 \pm 0.07^{\star}$ & $0.86 \pm 0.09^{\star \#}$ & $1.37 \pm 0.10$ & $0.93 \pm 0.08^{\star}$ & $0.94 \pm 0.16^{\star}$ \\
\hline Eicosadienoic acid C20:2 $\omega 6$ & $1.01 \pm 0.10$ & $0.15 \pm 0.01^{*}$ & $0.16 \pm 0.01^{\star \#}$ & $0.27 \pm 0.02$ & $0.20 \pm 0.02^{*}$ & $0.20 \pm 0.04^{\star}$ \\
\hline Dihomo- $\gamma$-linolenic acid C20:3 $\omega 6$ & $0.46 \pm 0.20$ & $0.58 \pm 0.30^{*}$ & $0.61 \pm 0.40^{\star \#}$ & $0.39 \pm 0.01$ & $0.39 \pm 0.04$ & $0.39 \pm 0.07$ \\
\hline Arachidonic acid C20:4心6c & $10.15 \pm 0.10$ & $8.67 \pm 0.09^{*}$ & $10.14 \pm 0.12^{\#}$ & $10.18 \pm 0.20$ & $8.76 \pm 0.35^{\star}$ & $9.27 \pm 0.44^{\star \#}$ \\
\hline Docosatetraenoic acid C22:4 $\omega 6$ & $3.27 \pm 0.02$ & $2.60 \pm 0.28^{*}$ & $2.64 \pm 0.20^{\star \#}$ & $3.48 \pm 0.02$ & $2.47 \pm 0.09^{*}$ & $3.11 \pm 0.56^{\#}$ \\
\hline Docosapentaenoic acid C22:5 $\omega 3$ & $2.71 \pm 0.09$ & $4.00 \pm 0.12^{*}$ & $3.35 \pm 0.10^{\star \#}$ & $0.78 \pm 0.10$ & $4.17 \pm 0.02^{*}$ & $6.99 \pm 0.04^{\star \#}$ \\
\hline Docosahexaenoic acid C22:6 $\mathbf{3}$ & $8.19 \pm 0.32$ & $6.35 \pm 0.23^{*}$ & $6.78 \pm 0.40^{\star \#}$ & $10.74 \pm 0.00$ & $12.17 \pm 1.69^{\star}$ & $13.98 \pm 0.9^{\star \#}$ \\
\hline Total & 26.83 & $23.13^{\star}$ & $24.27^{\star \#}$ & 27.31 & $31.00^{\star}$ & $34.88^{\star \#}$ \\
\hline \multicolumn{7}{|l|}{ SUMS AND RATIONS } \\
\hline PUFA/SFA & 0.63 & $0.62^{*}$ & $0.57^{\star \#}$ & 0.47 & $0.93^{*}$ & $0.99^{\star \#}$ \\
\hline$\omega 3$ & 11.36 & $10.93^{\star}$ & $10.47^{\star \#}$ & 12.01 & $18.64^{*}$ & $21.36^{\star \#}$ \\
\hline$\omega 6$ & 15.47 & $12.20^{*}$ & $13.80^{\star \#}$ & 15.30 & $12.36^{\star}$ & $13.52^{\star \#}$ \\
\hline$\omega 9$ & 0.20 & $0.22^{*}$ & $0.20^{\#}$ & 0.61 & $0.66^{\star}$ & $0.67^{*}$ \\
\hline$\omega 6 / \omega 3$ & 1.36 & $1.12^{*}$ & $1.32^{\star}$ & 1.27 & $0.67^{*}$ & $0.63^{\star \#}$ \\
\hline
\end{tabular}

Data expressed as mean \pm standard deviation. CG, Control Group; AO, Avocado Oil Group; AP, Avocado Pulp Group. *Vs. CG. " vs. AO. TO, at birth; T21, at weaning (21 days of life). Statistical test used was One way Anova, followed by Tukey with a $(p<0.05)$ level of significance.

AO offspring, in relation to the CG, presented early PG onset, and the appearance of CA, GN, and FFR $(p<0.05)$ (Table 5).

For the somatic indicators, the AP neonates presented anticipation in auditory conduction opening and epidermic hair appearance, yet delayed eruption of inferior incisors as compared to the CG $(p<0.05)$. The same group (AP) when compared to the AO group presented anticipated auditory conduit opening together with superior incisor eruption $(p<0.05)$. The neonates of the AO group presented auditory conduction opening and inferior incisor eruption delays when compared to the CG ( $p<$ 0.05) (Table 6).

\section{Behavioral Testing Open Field}

Open Field Habituation Test ambulatory analysis at 45 days (adolescent stage) presented differences between the first and second exposures, with a decrease in the ambulation parameter during the second exposure for all groups: CG (77. $50 \pm 5.75$ and $55.36 \pm 5.44)$, AO (129.92 \pm 11.16 and $55.75 \pm 5.44)$, and
$\operatorname{AP}(115.56 \pm 11.13$ and $56.25 \pm 5.10)(p<0.05)$ (Figure 5A). In the adult phase (T90) the same differences persisted, yet with ambulation exposure decreases in the CG (112.82 \pm 10.57 and $51.67 \pm 5.57)$, $\mathrm{AO}(95.83 \pm 8.77$ and $38.50 \pm 4.97)$, and $\mathrm{AP}(99.71$ \pm 9.09 and $60.50 \pm 5.95)(p<0.05)$ (Figure 5B).

\section{Object Recognition Test (ORT) Adolescent phase}

In the adolescent phase, the rate of new object exploration in the short term and in the long term tests was higher in the AO and AP groups, presenting higher exploration rates as compared to the CG $(p<0.05)$ (Figures 6A,B). The groups AO and AP presented greater time for the new object, relative to the familiar object, in both short and long periods ( $p<0.05$ ) (Figures 6C,D).

\section{Adulthood}

Adult offspring in the AP and AO groups also presented higher rates of new object exploration in the short and long term $(p<0.05)$ (Figures 7A,B). The groups AO and AP 
TABLE 4 | Composition of fatty acids present in the brain offspring (T45 and T90) of dams supplemented with oil and avocado pulp.

\begin{tabular}{|c|c|c|c|c|c|c|}
\hline Fatty acids & \multicolumn{6}{|c|}{ Groups } \\
\hline \multicolumn{7}{|l|}{ SATURATED } \\
\hline Myristic acid C14:0 & $0.12 \pm 0.04$ & $0.11 \pm 0.00$ & $0.12 \pm 0.01$ & $0.13 \pm 0.02$ & $0.13 \pm 0.02$ & $0.11 \pm 0.01$ \\
\hline Behenic acid C22:0 & $0.19 \pm 0.03$ & $0.24 \pm 0.02^{*}$ & $0.22 \pm 0.01^{*}$ & $0.25 \pm 0.02$ & $0.33 \pm 0.01^{*}$ & $0.27 \pm 0.05^{\#}$ \\
\hline Lignoceric acid C24:0 & - & $0.15 \pm 0.01$ & $0.16 \pm 0.01^{\#}$ & $0.19 \pm 0.01$ & $0.19 \pm 0.01$ & $0.19 \pm 0.01$ \\
\hline Total SAT & 32.34 & $30.68^{*}$ & $32.61^{\star \#}$ & 33.10 & $37.13^{\star}$ & $33.89 * \#$ \\
\hline \multicolumn{7}{|l|}{ MONOUNSATURATED } \\
\hline 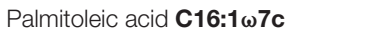 & $0.34 \pm 0.02$ & $0.30 \pm 0.05$ & $0.45 \pm 0.29$ & $0.30 \pm 0.03$ & $0.27 \pm 0.04$ & $0.25 \pm 0.07$ \\
\hline Total Monounsat & 18.69 & $18.36^{\star}$ & $19.68^{\star \#}$ & 22.43 & $25.11^{\star}$ & $23.52^{\star \#}$ \\
\hline \multicolumn{7}{|l|}{ POLYUNSATURATED } \\
\hline Linoleic acid C18:2 $\omega 6 \mathrm{c}$ & $0.59 \pm 0.13$ & $0.63 \pm 0.08$ & $0.61 \pm 0.11$ & $0.61 \pm 0.00$ & $0.67 \pm 0.04^{\star}$ & $0.53 \pm 0.06^{\star \#}$ \\
\hline Eicosadienoic acid C20:2 $\omega 6$ & $0.23 \pm 0.06$ & $0.22 \pm 0.00$ & $0.22 \pm 0.02$ & $0.22 \pm 0.01$ & $0.25 \pm 0.00^{*}$ & $0.12 \pm 0.02^{\star \#}$ \\
\hline Dihomo- $\gamma$-linolenic acid C20:3 $\omega 6$ & $0.35 \pm 0.07$ & $0.36 \pm 0.03$ & $0.39 \pm 0.00$ & $0.28 \pm 0.02$ & $0.36 \pm 0.01^{*}$ & $0.24 \pm 0.02^{\#}$ \\
\hline 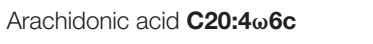 & $6.41 \pm 0.43$ & $7.09 \pm 0.52$ & $8.06 \pm 0.64^{\star \#}$ & $7.23 \pm 0.61$ & $8.17 \pm 0.74^{\star}$ & $7.59 \pm 0,10$ \\
\hline Docosatetraenoic acid C22:4 $\omega 6$ & $3.20 \pm 0.70$ & $3.17 \pm 1.02$ & $3.90 \pm 0.59$ & $2.74 \pm 0.23$ & $2.79 \pm 0.16$ & $2.80 \pm 0.18$ \\
\hline Docosapentaenoic acid C22:5 $\omega 3$ & $3.28 \pm 0,69$ & $4.60 \pm 0.89^{\star}$ & $3.28 \pm 0.85^{\#}$ & $1.43 \pm 0.13$ & $1.40 \pm 0.02$ & $1.40 \pm 0.09$ \\
\hline Docosahexaenoic acid C22:6 $\mathbf{3}$ & $11.39 \pm 1.37$ & $15.86 \pm 1.03^{*}$ & $16.76 \pm 1.52^{\star}$ & $11.45 \pm 1.24$ & $10.48 \pm 0.43$ & $15.80 \pm 0.97^{\star \#}$ \\
\hline Total & 25.45 & $31.93^{*}$ & $33.22^{\star \#}$ & 23.96 & $24.12^{\star}$ & $28.48^{\star \#}$ \\
\hline
\end{tabular}

Data expressed as mean \pm standard deviation. CG, Control Group; AO, Avocado oil Group; AP, Avocado Pulp Group of. "vs. CG. " vs. AO. T45, adolescent phase (45 days of life); T90, adult phase (90 days of life). Statistical test used was One Anova way followed by Tukey with a level of significance of $(p<0.05)$.

presented greater time for the new object, relative to the familiar object, in both short and long periods $(p<0.05)$ (Figures 7C,D).

\section{DISCUSSION}

Maternal fatty acids transferred via the placenta, and through the breast milk are considered critical for growth and development (Lauritzen and Carlson, 2011; Innis, 2014). Thus, during gestation and lactation, manipulation of lipids can affect the availability of fatty acids to the fetus and the infant. In the present study, avocado oil and pulp supplementation during gestation and lactation positively influenced the offspring in: (1) reflex development, (2) somatic maturation, (3) and memory acquisition (4) the fatty acid profiles of the brains of the neonates, adolescents, and adult offspring.
Maternal consumption of distinct lipids presents differing consequences for the weight, growth, and somatic parameters in their offspring. At birth, and during the first week of lactation, the results reveal that the offspring of the AP mothers had lower weights than the CG. Several studies have reported a decrease in the body weights of offspring with mothers receiving lipids from differing sources; at times presenting similar lipid profiles as compared to those used in the present research, such as cashew nuts (Melo et al., 2017), which has fiber as Avocado pulp and Buriti oil (Mauritia flexuosa) (Medeiros et al., 2015), and olive oil (Sánchez et al., 2012; Priego et al., 2013), which are source of polyunsaturated fatty acids as the lipids used in the present research (pulp and avocado oil). The presence of fiber and polyunsaturated fatty acids in maternal diet can induce reduction of plasma triglycerides (TG). Increased maternal levels have been used as a biochemical marker to increase offspring birth weight (Barbour and Hernandez, 
TABLE 5 | Reflex maturation in offspring of mothers supplemented with avocado oil and pulp during gestation and lactation.

\begin{tabular}{|c|c|c|c|}
\hline \multirow[b]{2}{*}{ Reflexes } & \multicolumn{3}{|c|}{ Groups } \\
\hline & CG & AO & AP \\
\hline Palmar grasp (PG) & $8(6-13)$ & $5(3-7)^{\star}$ & $4(3-5)^{\star}$ \\
\hline Righting reflex (RR) & $4(1-9)$ & $4(2-7)$ & $4(2-6)$ \\
\hline Vibrissa placing $(\mathrm{VP})^{\mathrm{b}}$ & $10(5-13)$ & $9(7-10)$ & $7(7-10)^{\star \#}$ \\
\hline Cliff avoidance $(C A)^{b}$ & $10(6-15)$ & $6(5-8)^{\star}$ & $6(2-10)^{\star}$ \\
\hline Negative geotaxis $(\mathrm{GN})^{\mathrm{b}}$ & $20(19-21)$ & $13(12-14)^{\star}$ & $10(10-12)^{\star \#}$ \\
\hline Auditory tartle response (AS) ${ }^{b}$ & $12(11-13)$ & $13(12-13)$ & $11(10-12)^{\star} \#$ \\
\hline Free-fall righting $(\mathrm{FFR})^{\mathrm{b}}$ & $12(8-15)$ & $7(2-14)^{\star}$ & $5(2-9)^{\star}$ \\
\hline
\end{tabular}

Data were expressed as mean values of the day (Min-Max) and analyzed by Kruskal-Wallis analysis of variance followed by Dunn's test $(p<0.05)$.

${ }^{\star}$ Compared to control group.

\#Compared to the avocado oil group.

Considering: a Day of response disappearance and ${ }^{b}$ Day of response appearance. CG (Control Group-n = 15), AO (Avocado Oil Group-n = 15), AP (Avocado Pulp Group$n=15)$.

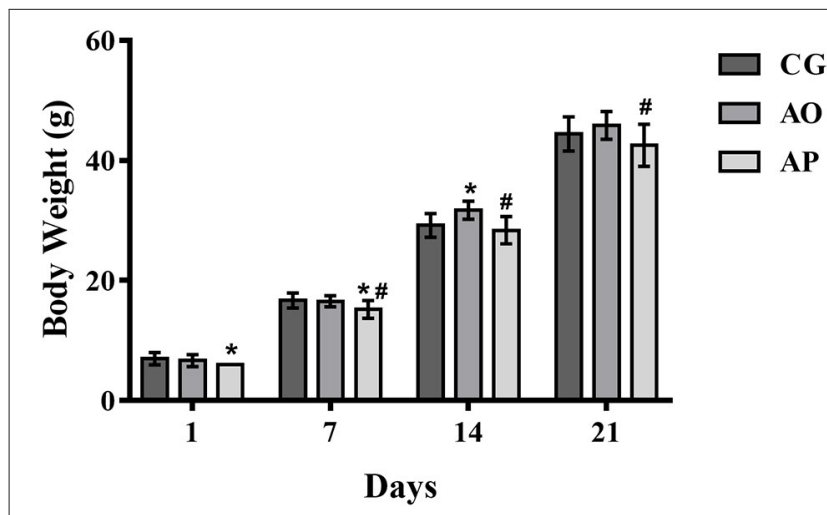

FIGURE 3 | Mean body weight in grams (g) ( \pm SEM) of neonatal rats whose mothers received supplementation with avocado oil and pulp (3,000 mg/kg body weight) during gestation and lactation. ANOVA followed by Tukey ( $p<$ 0.05). ( $\left.{ }^{\star}\right)$ statistically different as compared to CG; (\#) statistically different as compared to AO. CG (Control Group- $n=15)$, AO (Avocado Oil Group- $n=15)$, AP (Avocado Pulp Group- $n=15$ ).

2018) and their lower plasma levels have been associated with improved insulin sensitivity and lower caloric influx (Nolan et al., 1995). In contrast, diets with high levels of SFA induce an increase in plasma triglycerides, consequently, they also can induce increase in the offspring weight (Ferro Cavalcante et al., 2013; Soares et al., 2013; Cadena-Burbano et al., 2017). Therefore, it was observed in the present research a reduction in TG at the end of the lactation of the mothers fed with the pulp, when compared to the others groups (data not shown). These findings are in agreement with Barbour and Hernandez (2018).

Maternal supplementation with avocado promoted acceleration in the postnatal appearance of several somatic parameters. Lipids are recognized for promoting somatic growth in the offspring (Del Prado et al., 1997). Both avocado oil and

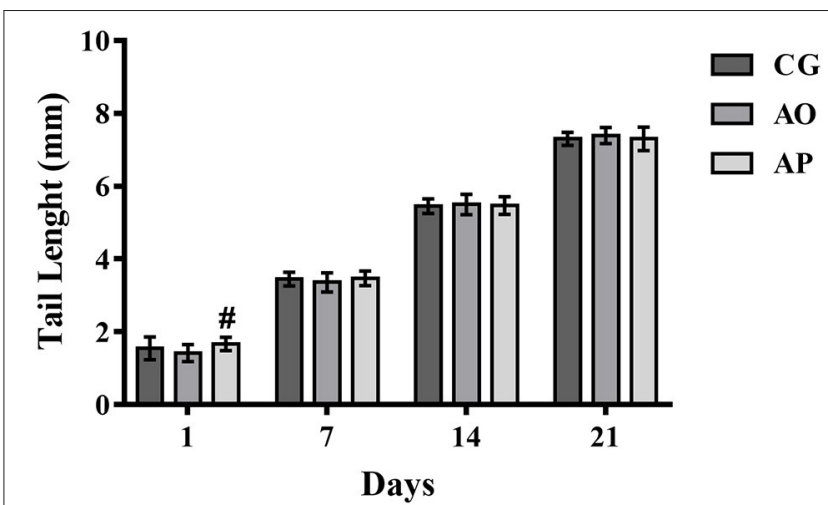

FIGURE 4 | Tail lengths of the offspring of mothers supplemented with avocado oil and pulp (3,000 mg/kg body weight) during gestation and lactation. Data expressed as mean \pm SEM and analyzed by ANOVA followed by Tukey $(p<0.05)$. (\#) statistically different as compared to the AO group. CG (Control Group- $n=15$ ), AO (Avocado Oil Group- $n=15$ ), AP (Avocado Pulp Group- $n=15)$.

TABLE 6 | Somatic development in offspring of mothers supplemented with avocado oil and pulp during gestation and lactation.

\begin{tabular}{lccc}
\hline & \multicolumn{3}{c}{ Groups } \\
\cline { 2 - 4 } Physical characteristics & CG & AO & AP \\
\hline Ear unfolding & $3(2-4)$ & $3(2-4)$ & $3(2-4)$ \\
Auditory conduit opening & $14(13-15)$ & $13(12-13)^{\star}$ & $11(10-12)^{\star \#}$ \\
Eye opening & $14(12-15)$ & $13(12-15)$ & $14(12-15)$ \\
Eruption of superior incisors & $10(8-12)$ & $11(9-12)$ & $9(8-11)^{\#}$ \\
Eruption of inferior incisors & $4(2-5)$ & $8(8-9)^{\star}$ & $7(7-8)^{\star}$ \\
Epidermic hair appearance & $3(2-4)$ & $3(3-3)$ & $3(2-3)^{\star}$
\end{tabular}

Data were expressed as mean values of the day (Min-Max), analyzed by Kruskal-Wallis analysis of variance, followed by Dunn's test $(p<0.05)$.

${ }^{*}$ Compared to the control group.

\# Compared to the avocado oil group.

CG (Control Group-n = 15), AO (Avocado Oil Group-n =15), AP (Avocado Pulp Group- $n=15$ ).

pulp, despite having high amounts of oleic and palmitoleic acids in their composition, have $\omega-6$ and $\omega-3$ fatty acids, which have been associated with physical growth in rat progeny (Santillán et al., 2010; Ferro Cavalcante et al., 2013). These results are consistent with experiments that used PUFA and MUFA in the source foods (Ferro Cavalcante et al., 2013; Melo et al., 2017), and the same for avocado lipids, yet results diverge for SFAsource diets (Soares et al., 2009). The findings confirm that both the quality and amount of lipids in the maternal diet directly influence physical development in the offspring (Hausman et al., 1991). DHA and ARA (in combination) are essential for optimal growth and development early in life (Harauma et al., 2017).

During the critical developmental phase, essential fatty acids are needed for physical growth and good brain development. The brain goes through processes that include neural network organization; accumulation of DHA and ARA occurs to support active neurogenesis and neuronal growth (Lauritzen and Carlson, 


\section{A} _T45 days of life

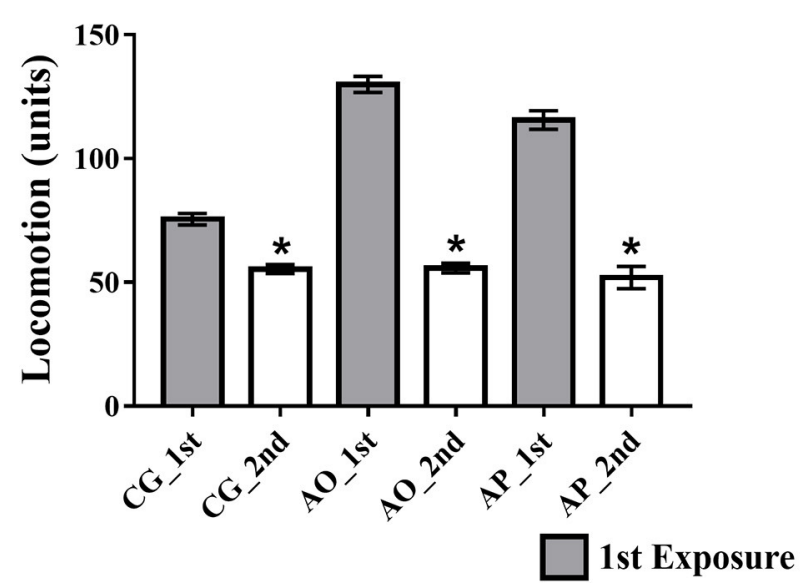

B

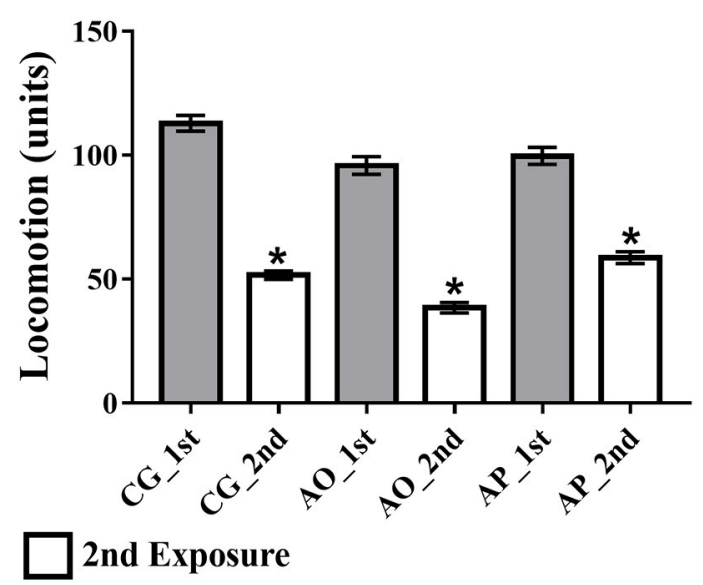

FIGURE 5 | Influence of maternal supplementation with avocado oil and pulp on total offspring ambulation. Data expressed as mean and standard error ( \pm SEM), analyzed by ANOVA, and followed by Tukey $(p<0.05)$. (A) Adolescent phase offspring (T45); (B) Adult offspring (T90). 1st: first exposure; 2nd: second exposure. CG (Control Group- $n=15$ ), AO (Avocado Oil Group- $n=15$ ), AP (Avocado Pulp Group- $n=15$ ). * $p<0.05$ vs. 1 st exposure in the open field.

2011; Innis, 2014), while modifying the fluidity and signaling of neuronal membranes (Bazinet and Layé, 2014). In this period, specific brain regions, including the hippocampus, striatum, visual and auditory cortices respond similarly to nutritional insults (Kretchmer et al., 1996), leading to long-term effects (Morgane et al., 1993; Arcego et al., 2017).

Our results showed that at the end of gestation, or at the beginning of the postnatal phase (T0), there was less incorporation of DHA $(\mathrm{C} 22: 6 \mathrm{n} 3)$ in the brains of the $\mathrm{AO}$ and AP offspring. However, by the end of lactation, levels of DHA had increased in brains of the AO and AP offspring as compared to the control groups. In rodents, fetal demand for fatty acid incorporation occurs from the last week of gestation to the end of lactation (Morgane et al., 2002). This explains the observed increase in DHA incorporation in the T21 brain levels, as compared to T0 levels. Accumulation of fatty acids in the offsprings' brains is influenced by pre-fetal and post-fetal maternal supply (Innis, 2011). Avocado oil and pulp present low linolenic acid content (ALA); a DHA precursor. The increases observed in the brain levels for this fatty acid in the offspring of mothers who consumed avocado oil and pulp oppose studies that have found a positive relation between low ALA content and low proportions of DHA in offspring brain tissue (Amusquivar et al., 2000; Melo et al., 2017; Lopez-Soldado et al., 2018). However, avocado presents high phospholipid (PL) content, present in the lipid fraction of its pulp (Cowan and Wolstenholme, 2016; Pacetti et al., 2017) and oil (Takenaga et al., 2008). Increases in DHA uptake in the brains of the AO and AP offspring can be explained by the presence of phospholipids in avocado. DHA is synthesized by ALA desaturation and stretching reactions (Pereira et al., 2003; Novak et al., 2008), and when esterified into PLs, is more efficiently incorporated into brain tissue (Murru et al., 2013; Kitson et al., 2016; Destaillats et al., 2018). Of the phospholipids, lyso-phosphatidylcholine (LPC) as esterified to DHA (LPC-DHA) is the most efficient way to cross the blood-brain barrier inducing a greater deposition of DHA in the brain (Nguyen et al., 2014). In the fetal brain formation and postnatal development periods, LPC-DHA is associated with an increase in exogenous PUFA uptake and deposition in the membranes of brain tissue, which promotes higher DHA deposition (Chan et al., 2018). One study reveals that offspring of mothers fed LPC from DHA-enriched eggs present higher levels of this FA in certain brain regions (Valenzuela et al., 2010). These findings are similar to the data found in the present study.

Reflex ontogeny is another parameter used to evaluate development because it measures maturation and central nervous system function early in life (Fox, 1965; Smart and Dobbing, 1971). It also reflects the integrity of cerebellar and sensorimotor development, and of vibrissae integration (Zhang et al., 2010). Adequate reflex development depends on myelination and synapse processes, and the action of neurotransmitters (Bourre et al., 1987; Morgane et al., 1993). The anticipation of the negative geotaxis demonstrates positive evolution in labyrinth and/or vestibule function, while anticipation of cliff avoidance reflects sensorimotor function maturity (Santillán et al., 2010). Righting reflex involves both motor and visual functions (Boyle, 2001) and confirms the nervous system's maturation. Our results showed that avocado oil and pulp promoted acceleration of neonate reflex maturation. A number of experimental studies support the results of the present study for animals treated with cashew nuts (Melo et al., 2017), soybean and fish oil (Santillán et al., 2010), and goat's milk fat (Soares et al., 2013). However, our results verify that consumption of avocado pulp promotes a more pronounced acceleration in reflex; by anticipating six of the seven observed parameters. At the end of lactation, SFA and MUFA levels were lower in the brains of the animals treated with oil and pulp than in the control group; while PUFA levels were higher in the 

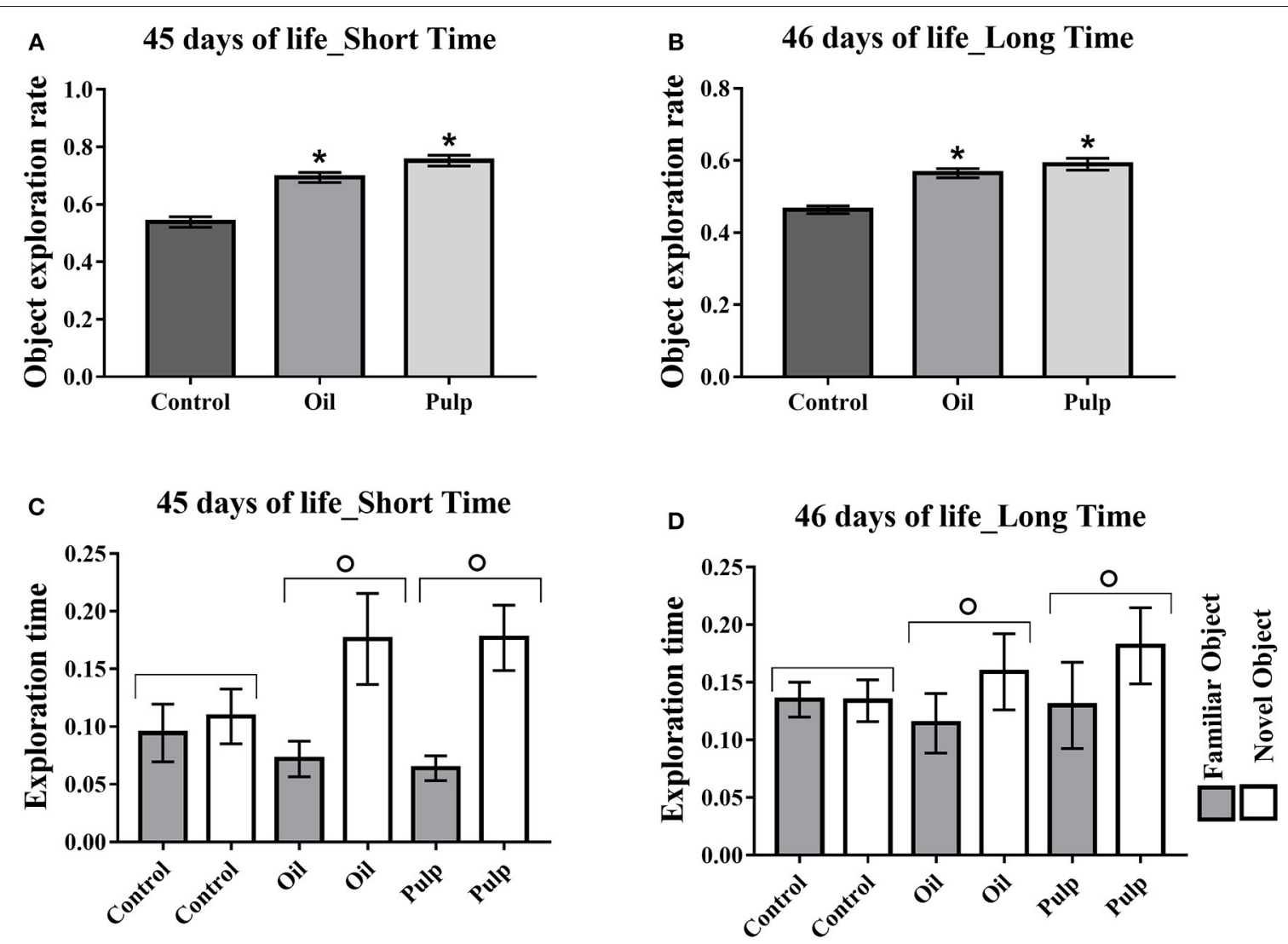

FIGURE 6 | Adolescent offspring; short and long term memory test; preference in the object exploration. Data expressed as mean and standard error ( \pm SEM) (A,B) and mean and standard deviation $( \pm S D)$ (C,D) Analyzed by ANOVA, and followed by Tukey $(p<0.05)$. (A) Object exploration rate short term. (B) Object exploration rate long term. (C) Time of exploration of the family object and new object in the short term. (D) Time of exploration of the familiar object and new object in the long term. *Indicates a significant difference between the AO and AP groups vs. the CG in new object exploration time ( $p<0.05)$. ${ }^{\circ}$ Indicates a significant difference for the same group, in the time of exploration of the familiar object and the new object. Control (Control Group- $n=15$ ), Oil (Avocado Oil Group- $n=15$ ), Pulp (Avocado Pulp Group- $n=15)$.

brains of the animals treated with oil (13.5\%) and pulp (28\%). These results suggest that high levels of PUFA may be directly related to the offspring's reflex development and the higher consumption of pulp justifies the better result observed in these groups.

The avocado used in the present research is a source of bioactive components such as phenolics, flavonoids, and carotenoids (Ameer, 2016), and the pulp has more of these compounds than the oil. These substances cross the placental barrier reaching the fetal tissue (Todaka et al., 2005), accumulating in the retina (carotenoids) and playing an important role in the development of vision and the nervous system (Hammond, 2015; Zielinska et al., 2017). Thus, neonate neuroprotection (polyphenols) (Loren et al., 2005) can induce acceleration of somatic development and reflex in the offspring (phenolics and flavonoids) (Ajarem et al., 2017). As well was observed in the present work, where both avocado oil and pulp promoted such acceleration in the development of the offspring; the results for pulp being more pronounced. An opposing result was found by Medeiros et al. (2015) D'avila et al. (2017), where the offspring of mothers supplemented with Buriti oil (rich in carotenoids), presented delayed onset for palm grasp, righting reflex and cliff avoidance reflexes.

In addition, we investigated long-lasting effects of maternal supplementation on adolescent (T45) and adult (T90) offspring, evaluating the influence of avocado consumption on animal memory. At different stages of the cycle, neurons are continuously produced in the dentate gyrus of the hippocampus, but the ontogenetic stage in which the neurogenesis occurs is crucial for memory processing. Neurons in the neonatal phase are activated through different memory processes (Tronel et al., 2015). Learning and memory processes are performed in the hippocampus dentate gyrus in cooperation with the cerebral cortex (Eichenbaum and Lipton, 2008; Coutureau and Di Scala, 2009), and PUFAs, through metabolic imprinting mechanisms affect brain functions during the development phase and promote permanent effects (van Dijk et al., 2011; Yehuda, 2012).

ARA and DHA are important constituents of membranes, especially brain tissue (Martinez, 1992; Innis, 2007) and are involved in different mechanisms that affect animal memory. 

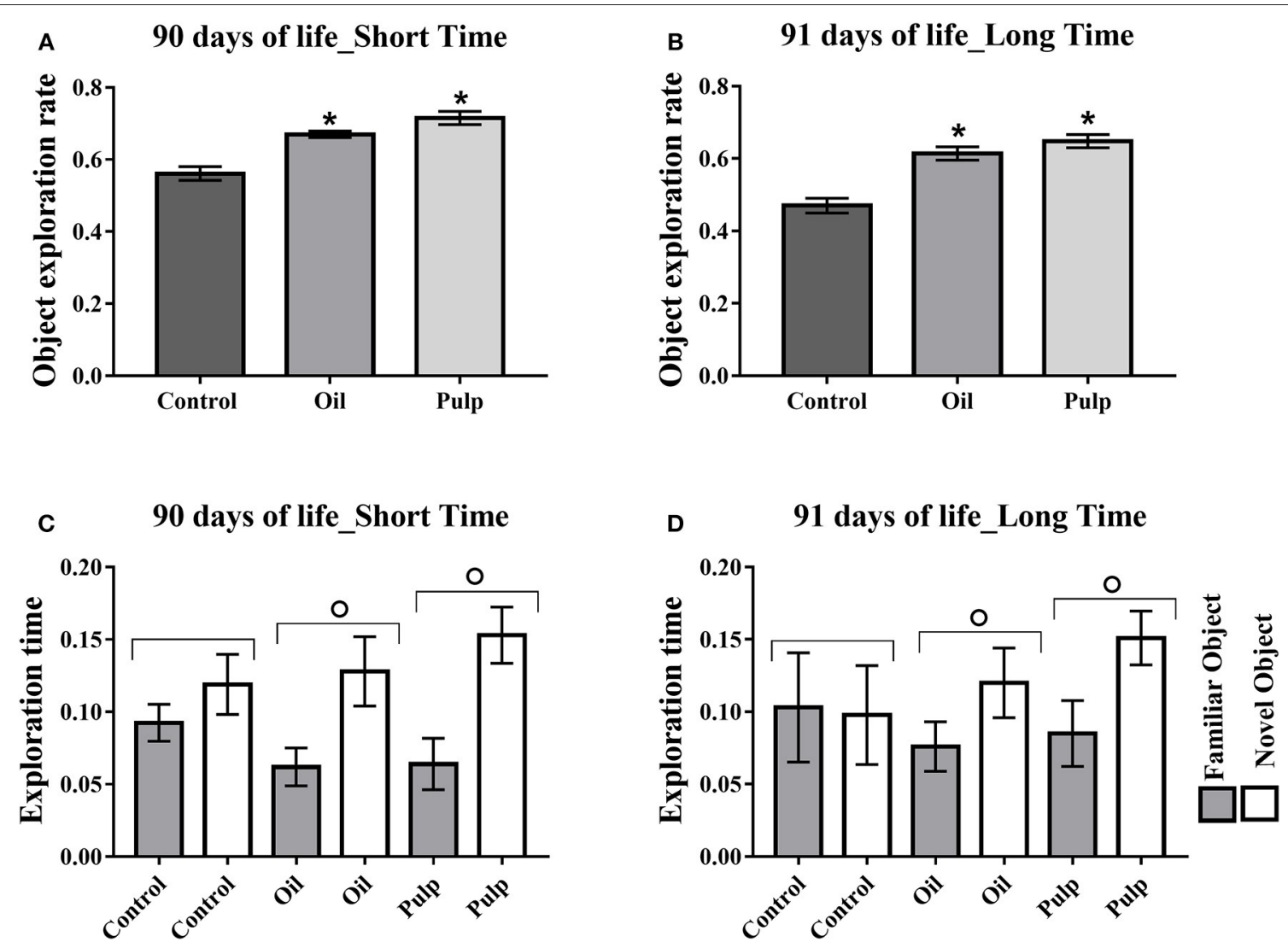

FIGURE 7 | Exploration of test objects (short and long-term memory) in adult offspring. Data expressed as mean and standard error of the mean ( \pm SEM), (A,B) and mean and standard deviation $( \pm S D)(\mathbf{C}, \mathbf{D})$ analyzed by ANOVA, followed by Tukey $(p<0.05)$. (A) Short term memory object exploration test. (B) Long-term memory object exploration test. (C) Time of exploration of the family object and new object in the short term. (D) Time of exploration of the familiar object and new object in the long term. *Indicates a significant difference between the AP and AO groups vs. the CG in new object exploration time. ${ }^{\circ}$ Indicates a significant difference for the same group, in the time of exploration of the familiar object and the new object. Control (Control Group- $n=15$ ), Oil (Avocado Oil Group- $n=15$ ), Pulp (Avocado Pulp Group- $n=15)$.

DHA is involved in the expression of BNDF (brain derived neurotrophic factor), NMDA receptor (N-methyl-D-aspartate) synthesis, induction of LTP (long-term potential), and liberating glutamate in glutamatergic functions. Deficiency of $\omega-3$ PUFA alters the fatty acid composition of the fetal brain with repercussions in the adult phase, increases fetal inflammatory processes, and induces deficits in development and memory (Labrousse et al., 2018). ARA is involved in the regulation of the cholinergic neurotransmission system and in the GABA/Glu regulatory system decreasing oxidative damage, and cellular apoptosis (Li et al., 2015). These two PUFAs were incorporated into the offsprings' brains through maternal supplementation with avocado, and ARA presented higher levels in the brains of the AO group in adolescence and the AP group as adults, while DHA presented higher levels in the AP and AO groups in adolescence and only in AP animals in adulthood.

In the present study we used the Open Field Habituation test and the Object Recognition Test (ORT) for evaluation of non-associative learning of the adolescent and adult offspring. In the Open Field Habituation test, repeated exposure to the same environment tends to cause a decrease in locomotion, recognized as a form of non-associative learning (Rachetti et al., 2012). Our results showed that in the adolescent and adult offspring, maternal supplementation with avocado oil and pulp reduced locomotion in the second exposure. The same effect has also observed in the animals fed a diet containing cashew nuts (Melo et al., 2017) and fish oil (Rachetti et al., 2012). Increased habituation, yet with memory impairment has occurred with peanut oil, containing little LA (Frances et al., 1996), but with an excess in saturated fat (Page et al., 2014).

The Object Recognition Test (ORT) involves an acquisition phase, where the rodent explores a chamber containing two similar objects and a recall phase, which occurs after a time interval in which one object is replaced by a new one. From the time interval used between the exposures, and from the ratio of time spent on the exploration of the new vs. the familiar object, and from the greater interaction with the new object, we may observe facilitation of short and long term memory (Cordner and Tamashiro, 2015); recognition of place, that involves the hippocampus (Barker and Warburton, 2011) and preference for 
the new object, which involves the prefrontal cortex (Mumby and Pinel, 1994; Bussey et al., 2000). Our results demonstrated that maternal supplementation with avocado oil and pulp facilitated acquisition of recognition memory in the adolescent and adult offspring, evidenced by a higher exploration rate (of the new object), both short and long term. Melo et al. (2017) has demonstrated that a maternal diet containing cashew nuts yields good short-term memory performance in the offspring. The offspring of mothers supplemented with fish oil has been shown to present good long-term memory performance (Rachetti et al., 2012). Other studies have demonstrated improvements in cognitive performance in offspring in relation to maternal consumption of olive (Pase et al., 2015) and linseed oils (Fernandes et al., 2011). However, maternal consumption of high $\omega-6 / \omega-3$ ratio (Lépinay et al., 2015); saturated fats (Frances et al., 1996; Souza et al., 2012; Arcego et al., 2017), hydrogenated vegetable fat (Pase et al., 2017) and interesterified fat (D'avila et al., 2017) caused damage to the animals' memory. The results obtained in our study demonstrate that increased ARA and DHA levels in the brains of the offspring of the supplemented groups interfered directly in memory development. Yet both DHA and ARA are responsible for maintaining optimal growth and functional behavior of the offspring (Harauma et al., 2017). DHA, in particular, is capable of protecting the hippocampus against oxidative stress and apoptosis; preventing memory deficits (Gao et al., 2016).

The positive effects of maternal supplementation with avocado oil and pulp on the memory of from adolescents to adult offspring can also be explained by its antioxidant potential. Experimentally, the antioxidant action of this fruit has been proven in diabetic rats supplemented with its oil (Ortiz-Avila et al., 2015). Studies have shown that the effect of maternal consumption of flavonoids on offspring memory is associated with decreased oxidative brain damage; due to reductions in lipid peroxidation levels, and generation of reactive species, and an increase in the antioxidant defense system as well as BDNF in the adult rat pre-frontal cortex (Bussey et al., 2000), and modulation of hippocampal signaling (Corona et al., 2013).

In the present study, the fatty acid profile in the brains of the offspring of mothers supplemented with avocado oil and pulp during gestation and lactation was measured at different

\section{REFERENCES}

Ajarem, J., Al Rashed, G., Mohany, M., and Allam, A. (2017). Neurobehavioral changes in mice offspring exposed to green tea during fetal and early postnatal development. Behav. Brain Funct. 13:10. doi: 10.1186/s12993-017-0128-1

Ameer, K. (2016). Avocado as a major dietary source of antioxidants and its preventive role in neurodegenerative diseases. Adv. Neurobiol. 12, 337-354. doi: 10.1007/978-3-319-28383-8_18

Amusquivar, E., Ruperez, F. J., Barbas, C., and Herrera, E. (2000). Low arachidonic acid rather than alpha-tocopherol is responsible for the delayed postnatal development in offspring of rats fed fish oil instead of olive oil during pregnancy and lactation. J. Nutr. 130, 2855-2865. doi: 10.1093/jn/130.11.2855

Apryatin, A. S., Sidorova, Y. S., Shipelin, V. A., Balakina, A., Trusov, N. V., and Mazo, V. K. (2017). Neuromotor activity, anxiety and cognitive function in the in vivo model of alimentary hyperlipidemia and obesity. Bull. Exp. Biol. Med. 163, 37-41. doi: 10.1007/s10517-017-3732-z stages of the life cycle. The animals of the two experimental groups, oil and pulp showed better somatic maturation, an anticipation of reflexes and improvement in memory. These findings demonstrate the benefits that maternal supplementation with a source of monounsaturated fatty acids and antioxidant compounds can bring to the development of the brain, persisting into adulthood.

As a limitation of this study, the groups treated with avocado were not compared with animals treated with lipid source deficient in essential fatty acids. On the other hand, we objected with the present study to define whether maternal supplementation with pulp and avocado oil could have a distinct effect on neurodevelopment of the offspring.

\section{CONCLUSION}

Maternal supplementation with avocado oil and pulp influences the development of the nervous system of the offspring in the short and long term, accelerating somatic development and reflex maturation while improving memory in the adolescent and adult phases.

\section{AUTHOR CONTRIBUTIONS}

This research was carried out by all authors. JS, MP, and MM designed the theme of the study. MM, RM, ES, DP, and MC performed the experimental methods designed. SS and CD performed fatty acid analysis and VV carried out analysis of the antioxidant components. JS, MO, FM, and MM analyzed the data. IS and MM interpreted the results and wrote the article.

\section{FUNDING}

This study was financed in part by the Coordenação de Aperfeiçoamento de Pessoal de Nível Superior-Brasil (CAPES)Finance Code 001.

\section{ACKNOWLEDGMENTS}

We thank all the contributing authors of the study. 
power and ascorbic acid concentration. Methods Enzymol. 299, 15-27. doi: 10.1016/S0076-6879(99)99005-5

Bourre, J. M., You You, A., Durand, G., and Pascal, G. (1987). Slow recovery of the fatty acid composition of sciatic nerve in rats fed a diet initiallylow in $\omega$-3fatty acids. Lipids 22, 535-538. doi: 10.1007/BF02540371

Boyle, R. (2001). Vestibulospinal control of reflex and voluntary head movement. Ann. NY. Acad. Sci. 942, 364-380. doi: 10.1111/j.1749-6632.2001.tb03760.x

Brenna, J. T., and Lapillonne, A. (2009). Background paper on fat and fatty acid requirements during pregnancy and lactation. Ann. Nutr. Metab. 55, 97-122. doi: $10.1159 / 000228998$

Bussey, T. J., Duck, J., Muir, J. L., and Aggleton, J. P. (2000). Distinct patterns of behavioural impairments resulting from fornix transection or neurotoxic lesions of the perirhinal and postrhinal cortices in the rat. Behav. Brain Res. 111, 187-202. doi: 10.1016/S0166-4328(00)00155-8

Cadena-Burbano, E. V., Cavalcanti, C. C. L., Lago, A. B., Benjamim, R. A. C., Oliveira, T. R. D. P., Silva, J. M., et al. (2017). A maternal highfat/high-caloric diet delas reflex ontogeny during lactation but enhances locomotor performance during late adolescence in rats. Nutr. Neurosci. 28, 1-12. doi: 10.1080/1028415X.2017.1354958

Chan, J. P., Wong, B. H., Chin, C. F., Galam, D. L. A., Foo, J. C., Wong, L. C., et al. (2018). The lysolipid transporter Mfsd2a regulates lipogenesis in the developing brain. PLoS Biol. 16:e2006443. doi: 10.1371/journal.pbio.2006443

Cordner, Z. A., and Tamashiro., K. L. K. (2015). Effects of high-fat diet exposure on learning \& memory. Physiol. Behav. 152, 363-371. doi: 10.1016/j.physbeh.2015.06.008

Corona, G., Vanzour, D., Hercelin, J., Williams, C. M., and Spencer, J. P. (2013). Phenolic acid intake, delivered via moderate champagne wine consumption, improves spatial working memory via the modulation of hippocampal and cortical protein expression/activation. Antiox. Redox Signal. 10, 1676-1689. doi: 10.1089/ars.2012.5142

Coutureau, E., and Di Scala, G. (2009). Entorhinal cortex and cognition. Prog. Neuropsychopharmacol. Biol. Psychiatry 33, 753-761. doi: $10.1016 /$ j.pnpbp.2009.03.038

Cowan, A. K., and Wolstenholme, B. N. (2016). “Avocado," in Encyclopedia of Food and Helth, eds B. Caballero, P. Finglas, F. Toldra (San Diego, CA: Academic Press), 294-299. doi: 10.1016/B978-0-12-384947-2.00049-0

D’avila, L. F., Dias, V. T., Vey, L. T., Milanesi, L. H., Roversi, K., Emanuelli, T., et al. (2017). Toxicological aspects of interesterified fat: brain damages in rats. Toxicol. Lett. 5, 122-128. doi: 10.1016/j.toxlet.2017.05.020

Del Prado, M., Delgado, G., and Villalpando, S. (1997). Maternal lipid intake during pregnancy and lactation alters milk composition and production and litter growth in rats. J. Nutr. 127, 458-462. doi: 10.1093/jn/127.3.458

Destaillats, F., Oliveira, M., Bastic Schmid, V., Masserey-Elmelegy, I., Giuffrida, F., Thakkar, S. K., et al. (2018). Comparison of the incorporation of DHA in circulatory and neural tissue when provided as Triacylglycerol (TAG), Monoacylglycerol (MAG) or phospholipids (PL) provides new insight into fatty acid bioavailability. Nutrients 10:E620. doi: 10.3390/nu10050620

Dreher, M. L., and Davenport, A. J. (2013). Hass avocado composition and potential health effects. Crit.Rev. Food. Sci. Nutr. 53, 738-750. doi: 10.1080/10408398.2011.556759

Dyall, S. C. (2017). Interplay between n-3 and n-6 long-chain polyunsaturated fatty acids and the endocannabinoid system in brain protection and repair. Lipids 52, 885-900. doi: 10.1007/s11745-017-4292-8

Eichenbaum, H., and Lipton, P. A. (2008). Towards a functional organization of the medial temporal lobe memory system: role of the parahippocampal and medial entorhinal cortical areas. Hippocampus 18, 1314-1324. doi: 10.1002/hipo.20500

Fernandes, F. S., Souza, A. S., Carmo, M. D., and Boaventura, G. T. (2011). Maternal intake of flaxseed-based diet (Linum usitatissimum) on hippocampus fatty acid profile: implications for growth, locomotor activity and spatial memory. Nutrition 27, 1040-1047. doi: 10.1016/j.nut.2010.11.001

Ferro Cavalcante, T. C., Lima da Silva, J. M., da Marcelino da Silva, A. A., Muniz, G. S., da Luz Neto, L. M., Lopes de Souza, S., et al. (2013). Effects of a westernized diet on the reflexes and physical maturation of male rat offspring during the perinatal period. Lipids 48, 1157-1168. doi: 10.1007/s11745-013-3833-z

Folch, J., Lees, M., and Sloane Stanley, G. H. (1957). A simple method for the isolation and purification of total lipids. J. Biol. Chem. 226, 497-509.

Fox, W. M. (1965). Reflex-ontogeny and behavioural development of the mouse. Anim. Behav. 13, 234-241. doi: 10.1016/0003-3472(65)90041-2
Frances, H., Monier, C., Clement, M., Lecorsier, A., Debray, M., and Bourre, J. M. (1996). Effect of dietary alpha-linolenic acid deficiency on habituation. Life Sci. 58, 1805-1816. doi: 10.1016/0024-3205(96)00164-6

Gao, J., Wu, H., Cao, Y., Liang, S., Sun, C., Wang, P., et al. (2016). Maternal DHA supplementation protects rat offspring against impairment of learning and memory following prenatal exposure to valproic acid. J. Nutr. Biochem. 35, 87-95. doi: 10.1016/j.jnutbio.2016.07.003

Garbay, B., Heape, A. M., Sargueil, F., and Cassagne, C. (2000). Myelin synthesis in the peripheral nervous system. Prog. Neurobiol. 61, 267-304. doi: 10.1016/S0301-0082(99)00049-0

González, H. F., and Visentin, S. (2016). Nutrients and neurodevelopment: lipids. Arch. Argent. Pediatr. 114, 472-476. doi: 10.5546/aap.2016.eng.472

Gustavsson, M., Hodgkinson, S. C., Fong, B., Norris, C., Guan, J., Krageloh, C. U., et al. (2010). Maternal supplementation with a complex milk lipid mixture during pregnancy and lactation alters neonatal brain lipid composition but lacks effect on cognitive function in rats. Nutr. Res. 30, 279-289. doi: 10.1016/j.nutres.2010.04.005

Hammond, B. R. (2015). Dietary carotenoids and the nervous system. Foods 4, 698-701. doi: 10.3390/foods4040698

Harauma, A., Hatanaka, E., Yasuda, H., Nakamura, M. T., Salem, N., and Moriguchi, T. (2017). Effects of arachidonic acid, eicosapentaenoic acid and docosahexaenoic acid on brain development using artificial rearing of delta-6desaturase knockout mice. Prostaglandins Leuk. Essent. Fatty Acids 127, 32-39. doi: $10.1016 /$ j.plefa.2017.10.001

Hartman, L., and Lago, R. C. A. (1973). Rapid preparation of fatty acids methyl esters. Lab. Pract. 22, 475-476.

Hausman, D. B., Mccloskey, H. M., and Martin, R. J. (1991). Maternal dietary fat type influences the growth and fatty acid composition of newborn and weanling rats. J. Nutr. 121, 1917-1923. doi: 10.1093/jn/121.12.1917

Herrera, E., and Ortega-Senovilla, H. (2014). Lipid metabolism during pregnancy and its implications for fetal growth. Curr. Pharm. Biotechnol. 15, 24-31. doi: 10.2174/1389201015666140330192345

Innis, S. M. (2004). Polyunsaturated fatty acids in human milk: an essential role in infant development. Adv. Exp. Med. Biol. 554, 27-43. doi: 10.1007/978-1-4757-4242-8_5

Innis, S. M. (2005). Essential fatty acid transfer and fetal development. Placenta 26, S70-S75. doi: 10.1016/j.placenta.2005.01.005

Innis, S. M. (2007). Fatty acids and early human development. Early Hum. Dev. 83, 761-766. doi: 10.1016/j.earlhumdev.2007.09.004

Innis, S. M. (2011). Metabolic programming of long-term outcomes due to fatty acid nutrition in early life. Matern. Child. Nutr. 7, S112-S123. doi: $10.1111 /$ j.1740-8709.2011.00318.x

Innis, S. M. (2014). Impact of maternal diet on human milk composition and neurological development of infants. Am. J. Clin. Nutr. 99, 734S-41S. doi: 10.3945/ajcn.113.072595

Kitson, A. P., Metherel, A. H., Chen, C. T., Domenichiello, A. F., Trépanier, M. O., Berger, A., et al. (2016). Effect of dietary docosahexaenoic acid (DHA) in phospholipids or triglycerides on brain DHA uptake and accretion. J. Nutr. Biochem. 33, 91-102. doi: 10.1016/j.jnutbio.2016.02.009

Kretchmer, N., Beard, J. L., and Carlson, S. (1996). The role of nutrition development of normal cognition. Am. J. Clin. Nutr. 63, 997-1001. doi: 10.1093/ajcn/63.6.997

Labrousse, V. F., Leyrolle, Q., Amadieu, C., Aubert, A., Serea, A., Coutureaud, E., et al. (2018). Dietary omega-3 deficiency exacerbates inflammation and reveals spatial memory deficits in mice exposed to lipopolysaccharide during gestation. Brain Behav. Immun. 73, 427-440. doi: 10.1016/j.bbi.2018.06.004

Lauritzen, L., and Carlson, S. E. (2011). Maternal fatty acid status during pregnancy and lactation and relation to newborn and infant status. Matern. Child. Nutr. 7, 41-58. doi: 10.1111/j.1740-8709.2011.00303.x

Lépinay, A. L., Larrieu, T., Joffre, C., Acar, N., Gárate, I., Castanon, N., et al. (2015). Perinatal high-fat diet increaseshippocampal vulnerability to the adverseeffects of subsequent high-fat feeding. Psychoneuroendocrinology 53, 82-93. doi: 10.1016/j.psyneuen.2014.12.008

Li, C., Wang, Q., Li, L., Liu, Y., and Diao, H. (2015). Arachidonic acid attenuates learning and memory dysfunction induced by repeated isoflurane anesthesia in rats. Int. J. Clin. Exp. Med. 8, 12365-12373.

Liu, M., Li, X. Q., Weber, C., Lee, C. Y., Brown, J., and Liu, R. H. (2002). Antioxidant and antiproliferative activities of 
raspberries. J. Agric. Food. Chem. 50, 2926-2930. doi: 10.1021/jf01 11209

Lopez-Soldado, I., Ortega-Senovilla, H., and Herrera, E. (2018). Maternal adipose tissue becomes a source of fatty acids for the fetus in fasted pregnant rats given diets with different fatty acid compositions. Eur. J. Nutr. 57, 2963-2974. doi: 10.1007/s00394-017-1570-4

Loren, D. J., Seeram, N. P., Schulman, R. N., and Holtzman, D. M. (2005). Maternal dietary supplementation with pomegranate juice is neuroprotective in an animal model of neonatal hypoxic-ischemic brain injury. Pediatr. Res. 57, 858-864. doi: 10.1203/01.PDR.0000157722.07810.15

Makrides, M., Collins, C. T., and Gibson, R. A. (2011). Impact of fatty acid status on growth and neurobehavioural development in humans. Matern. Child. Nutr. 7, 80-88. doi: 10.1111/j.1740-8709.2011.00304.x

Martinez, M. (1992). Tissue levels of polyunsaturated fatty acids during early human development. J. Pediatr. 120, 129S-138S. doi: 10.1016/S0022-3476(05)81247-8

Medeiros, M. C., Aquino, J. S., Soares, J., Figueiroa, E. B., Mesquita, H. M., Pessoa, D. C., et al. (2015). Buriti oil (Mauritia flexuosa L.) negatively impacts somatic growth andreflex maturation and increases retinol deposition in young rats. Int. J. Dev. Neurosci. 46, 7-13. doi: 10.1016/j.ijdevneu.2015.05.001

Medina, J. M., and Tabernero, A. (2002). Astrocyte-synthesized oleic acid behaves as a neurotrophic factor for neurons. J. Physiol. Paris 96, 265-271. doi: 10.1016/S0928-4257(02)00015-3

Melo, M. F. F. T., Pereira, D. E., Sousa, M. M., Medeiros, D. M. F., Lemos, L. T. M., Madruga, M. S., et al. (2017). Maternal intake of cashew nuts accelerates reflex maturation and facilitates memory in the offspring. Int. J. Dev. Neurosci. 61, 58-67. doi: 10.1016/j.ijdevneu.2017.06.006

Mennitti, L. V., Oliveira, J. L., Morais, C. A., Estadella, D., Oyama, L. M., Oller do Nascimento, C. M., et al. (2015). Type of fatty acids in maternal diets during pregnancy and/or lactation and metabolic consequences of the offspring. $J$. Nutr. Biochem. 26, 99-111. doi: 10.1016/j.jnutbio.2014.10.001

Morgane, J. P., Mokler, D. J., and Galler, J. R. (2002). Effects of prenatal protein malnutrition on the hippocampal formation. Neurosci. Biobehav. Rev. 26, 471-483. doi: 10.1016/S0149-7634(02)00012-X

Morgane, P. J., Austin-LaFrance, R., Bonzio, J., Tonkiss, J., Díaz-Cintra, S., Cintra, L., et al. (1993). Prenatal malnutrition and development of the brain. Neurosc. Biobehav. Rev. 17, 91-128. doi: 10.1016/S0149-7634(05)80234-9

Mumby, D. G., and Pinel, J. P. (1994). Rhinal cortex lesions and object recognition in rats. Behav. Neurosci. 108, 11-18. doi: 10.1037/0735-7044.108.1.11

Murru, E., Banni, S., and Carta, G. (2013). Nutritional properties of dietary omega-3-enriched phospholipids. Biomed. Res. Int. 2013:965417. doi: 10.1155/2013/965417

Nava-Mesa, M. O., Lamprea, M. R., and Múnera, A. (2013). Divergent short- and long-term effects of acute stress in object recognition memory are mediated by endogenous opioid system activation. Neurobiol. Learn. Mem. 106, 185-192. doi: 10.1016/j.nlm.2013.09.002

Nguyen, L. N., Ma, D., Shu, G., Wong, P., Cazenave-Gassiot, A., Zhang, X., et al. (2014). Mfsd2a is a transporter for the essential omega-3 fatty acid docosahexaenoic acid. Nature 509, 503-506. doi: 10.1038/nature13241

Nolan, C. J., Riley, S. F., Sheedy, M. T., Walstab, J. E., and Beischer, N. A. (1995). Maternal serum triglyceride, glucose tolerance, and neonatal birth weight ratio in pregnancy. Diabetes Care 18, 1550-1556. doi: 10.2337/diacare.18.12. 1550

Novak, E. M., Dyer, R. A., and Innis, S. M. (2008). High dietary $\omega-6$ fatty acids contribute to reduced docosahexaenoic acid in the developing brain and inhibits secondary neurite growth. Brain Res. 1237, 136-145. doi: 10.1016/j.brainres.2008.07.107

Ortiz-Avila, O., Esquivel-Martínez, M., Olmos-Orizaba, B. E., Saaveedra-Molina, A., Rodriguez-Orozco, A. R., and Cortés-Rojo, C. (2015). Avocado oil improves mitochondrial function and decreases oxidative stress in brain of diabetic rats. J. Diabetes Res. 2015:485759. doi: 10.1155/2015/485759

Pacetti, D., Boselli, E., Lucci, P., and Frega, N. G. (2017). Simultaneous analysis of glycolipids and phospholids molecular species in avocado fruit. J. Chromatogr. A 1150, 241-251. doi: 10.1016/j.chroma.2006.10.022

Page, K. C., Jones, E. K., and Anday, E. K. (2014). Maternal and postweaning high-fat diets disturb hippocampal gene expression, learning, and memory function. Am. J. Physiol. Regul. Integr. Comp. Physiol. 306, R527-R537. doi: 10.1152/ajpregu.00319.2013
Pase, C. S., Roversi, K., Roversi, K., Vey, L. T., Dias, V. T., Veit, J. C., et al. (2017). Maternal trans fat intake during pregnancy or lactation impairs memory and alters BDNF and TrkB levels in the hippocampus of adult offspring exposed to chronic mild stress. Physiol. Behav. 169, 114-123. doi: 10.1016/j.physbeh.2016.11.009

Pase, C. S., Teixeira, A. M., Roversi, K., Dias, V. T., Calabrese, F., Molteni, R., et al. (2015). Olive oil-enriched diet reduces brain oxidative damages and ameliorates neurotrophic factor gene expression in diferente life stages of rats. J. Nutr. Biochem. 26, 1200-1207. doi: 10.1016/j.jnutbio.2015.05.013

Pereira, S. L., Leonard, A. E., and Mukerji, P. (2003). Recent advances in the study of fatty acid desaturases from animals and lower eukaryotes. Prostaglandins Leukot. Essent. Fatty Acids 68, 97-106. doi: 10.1016/S0952-3278(02)00259-4

Prado, E. L., Ashorn, U., Phuka, J., Maleta, K., Sadalaki, J., Oaks, B. M., et al. (2018). Associations of maternal nutrition during pregnancy and post-partum with maternal cognition and caregiving. Matern. Child. Nutr. 14:e12546. doi: $10.1111 / \mathrm{mcn} .12546$

Priego, T., Sánchez, J., García, A. P., Palou, A., and Pic,ó, C. (2013). Maternal dietary fat affects milk fatty acid profile and impacts on weight gain and thermogenic capacity of suckling rats. Lipids 48, 481-495. doi: 10.1007/s11745-013-3764-8

Pulido, R., Bravo, L., and Saura-Calixto, F. (2000). Antioxidant activity of dietary polyphenols as determined by a modified ferric reducing/antioxidant power assay. J. Agric. Food Chem. 48, 396-402. doi: 10.1021/jf9913458

Rachetti, A. L. F., Arida, R. M., Patti, C. L., Zanin, K. A., Fernades Santos, L., Frussa-Filho, R., et al. (2012). Fish oil supplementation and physical exercise program: distinct effects on different memory tasks. Behav. Brain. Res. 237, 283-289. doi: 10.1016/j.bbr.2012.09.048

Sánchez, J., Priego, T., García, A. P., Llopis, M., Palou, M., Pic,ó, C., et al. (2012). Maternal supplementation with an excess of different fat sources during pregnancy and lactation differentially affects feeding behavior in offspring: putative role of the leptin system. Mol. Nutr. Food. Res. 56, 1715-1728. doi: $10.1002 / \mathrm{mnfr} .201200211$

Santillán, M. E., Vincenti, L. M., Martini, A. C., Cuneo, M. F., Ruiz, R. D., Mangeaud, A., et al. (2010). Developmental and neurobehavioral effects of perinatal expo- sure to diets with different n-6: n-3 ratios in mice. Nutrition 26, 423-431. doi: 10.1016/j.nut.2009.06.005

Sinclair, A. J. (1975). Long-chain polyunsaturated fatty acids in the mammalian brain. Proc. Nutr. Soc. 34, 287-291. doi: 10.1079/PNS19750051

Smart, J. L., and Dobbing, J. (1971). Vulnerability of developing brain II. Effects of early nutritional deprivation on reflex ontogeny and development of behaviour in the rat. Brain Res. 28, 85-95. doi: 10.1016/0006-8993(71) 90526-9

Soares, A. K., Guerra, R. G., de Castro, M. L., Amancio-dos-Santos, A., Guedes, R. C. A., Cabral-Filho, J. E., et al. (2009). Somatic and reflex development in suckling rats: effects of mother treatment with ketogenic diet associated with lack of protein. Nutr. Neurosci. 12, 260-266. doi: 10.1179/147683009X4 23427

Soares, J. K. B., de Melo, A. P., Medeiros, M. C., Queiroga, R. C. E., Bomfim, M. A., Santiago, E. C., et al. (2013). Anxiety behavior is reduced, and physical growth is improved in theprogeny of rat dams that consumed lipids from goat milk: an elevatedplus maze analysis. Neurosc. Lett. 552, 25-29. doi: 10.1016/j.neulet.2013.07.028

Souza, A. S., Rocha, M. S., and Tavares do Carmo, M. D. (2012). Effects of a normolipidic diet containing trans fatty acids during perinatal period on the growth, hippocampus fatty acid profile, and memory of young rats according to sex. Nutrition 28, 458-464. doi: 10.1016/j.nut.2011.08.007

Surveswaran, S., Cai, Y. Z., Corke, H., and Sun, M. (2007). Systematic evaluation of natural phenolic antioxidants from 133 Indian medicinal plants. Food Chem. 102, 938-953. doi: 10.1016/j.foodchem.2006.06.033

Takenaga, F., Matsuyama, K., Abe, S., Torii, Y., and Itoh, S. (2008). Lipid and fatty acid composition of mesocarp and seed of avocado fruits harvested at northern range in Japan. J. Oleo Sci. 57, 591-597. doi: 10.5650/jos.57.591

Todaka, E., Sakurai, K., Fukata, H., Miyagawa, H., Uzuki, M., Omori, M., et al. (2005). Fetal exposure to phytoestrogens-The difference in phytoestrogen status between mother and fetus. Environ. Res. 99, 195-203. doi: 10.1016/j.envres.2004.11.006

Tronel, S., Lemaire, V., Charrier, V., Montaron, M. F., and Abrours, D. N. (2015). Influence of ontogenetic age on the role of dentate 
granule neuron. Brain Struct. Funct. 220, 645-661. doi: 10.1007/s00429-014$0715-y$

USDA (U.S. Department of Agriculture) (2011). Avocado, Almond, Pistachio and Walnut Composition. Nutrient Data Laboratory. USDA National Nutrient Database for Standard Reference, Release 24. Washington, DC: U.S. Department of Agriculture.

Valenzuela, A., Nieto, S., Sanhuenza, J., Morgado, N., Rojas, I., and Zañartu, P. (2010). Supplementing female rats with DHA-lysophosphatidylcholine increases docosahexaenoic acid and acetylcholine in the brain and improves the memory and learning capabilities of the pups. Grasas Aceites 61, 16-23. doi: 10.3989/gya.053709

van Dijk, G., Kacsándi, A., Kóbor-Nyakas, D. E., Hogyes, E., and Nyakas, C. (2011). Perinatal polyunstaurated fatty acids supplementation causes alterations in fuel homeostasis in adult male rats but does not offer resistance against STZ-induced diabetes. Horm. Metab. Res. 43, 938-943. doi: 10.1055/s-0031-12 91334

Yehuda, S. (2012). Polyunsaturated fatty acids as putative cognitive enhancers. Med. Hypotheses 79, 456-461. doi: 10.1016/j.mehy.2012.06.021

Zhang, Y., Li, N., Yang, J., Zhang, T., and Yang, Z. (2010). Effects of maternal food restriction on physical growth and neurobehavior in newborn wistar rats. Brain. Res. Bull. 83, 1-8. doi: 10.1016/j.brainresbull.2010. 06.005

Zhishen, J., Mengcheng, T., and Jianming, W. (1999). The determination of flavonoid contents in mulberry and their scavenging effects on superoxide radicals. Food Chem. 64, 555-559. doi: 10.1016/S0308-8146(98)00102-2

Zielinska, M. A., Wesołowska, A., Pawlus, B., and Hamułka, J. (2017). Health effects of carotenoids during pregnancy and lactation. Nutrients 9:E838. doi: $10.3390 /$ nu9080838

Conflict of Interest Statement: The authors declare that the research was conducted in the absence of any commercial or financial relationships that could be construed as a potential conflict of interest.

Copyright (c) 2019 Melo, Pereira, Moura, Silva, Melo, Dias, Silva, Oliveira, Viera, Pintado, Santos and Soares. This is an open-access article distributed under the terms of the Creative Commons Attribution License (CC BY). The use, distribution or reproduction in other forums is permitted, provided the original author(s) and the copyright owner(s) are credited and that the original publication in this journal is cited, in accordance with accepted academic practice. No use, distribution or reproduction is permitted which does not comply with these terms. 